\title{
A Global Lipschitz Continuity Result for a Domain Dependent Dirichlet Eigenvalue Problem for the Laplace Operator
}

\author{
Pier Domenico Lamberti and Massimo Lanza de Cristoforis
}

\begin{abstract}
Let $\Omega$ be an open connected subset of $\mathbb{R}^{n}$ for which the Poincaré inequality holds. We consider the Dirichlet eigenvalue problem for the Laplace operator in the open subset $\phi(\Omega)$ of $\mathbb{R}^{n}$, where $\phi$ is a locally Lipschitz continuous homeomorphism of $\Omega$ onto $\phi(\Omega)$. Then we show Lipschitz type inequalities for the reciprocals of the eigenvalues of the Rayleigh quotient
\end{abstract}

$$
\frac{\int_{\phi(\Omega)}|D v|^{2} d y}{\int_{\phi(\Omega)}|v|^{2} d y}
$$

upon variation of $\phi$, which in particular yield inequalities for the proper eigenvalues of the Dirichlet Laplacian when we further assume that the imbedding of the Sobolev space $W_{0}^{1,2}(\Omega)$ into the space $L^{2}(\Omega)$ is compact. In this case, we prove the same type of inequalities for the projections onto the eigenspaces upon variation of $\phi$.

Keywords: Dirichlet eigenvalues and eigenvectors, Laplace operator, domain perturbation, special nonlinear operators

MSC 2000: 35P15, 47H30

\section{Introduction}

In this paper, we plan to prove Lipschitz type estimates for the dependence of the reciprocals of the Dirichlet eigenvalues of the Rayleigh quotient and for the dependence of the projection onto the Dirichlet eigenspaces of the Laplace operator upon domain perturbation (cf. Theorems 3.15, 3.21). Our estimates apply for example to the general case of domains of finite measure, with no

P. D. Lamberti: Dipartimento di Matematica Pura ed Applicata, Università di Padova Via Belzoni 7, 35131 Padova, Italy; e-mail lamberti@math.unipd.it M. Lanza de Cristoforis: Dipartimento di Matematica Pura ed Applicata, Università di Padova Via Belzoni 7, 35131 Padova, Italy; e-mail mldc@math.unipd.it

ISSN 0232-2064 / \$ 2.50 (c) Heldermann Verlag Berlin 
regularity assumptions on the boundary. However, our methods allow us to handle an even more general class of domains without any further difficulty (cf. Theorems 3.3, 3.4, 3.15, 3.21.)

Spectral stability results concerning the Laplace operator in domain perturbation problems have been obtained by many authors (see Burenkov and Davies [2], Courant and Hilbert [3], Cox [4], Henry [8], Kato [9, pp. 423-426], Prodi [13], Sokolowski and Zolesio [16]).

To prove our inequalities, we need to prove some results in an abstract setting. Let $(H,<\cdot, \cdot>)$ be a real Hilbert space, which we shall consider as the 'environment' space. Then we consider a variable scalar product $Q$ on $H$, and we denote by $H_{Q}$ the linear space $H$ endowed with the scalar product $Q$, and by $\|\cdot\|_{Q}$ the norm associated to $Q$. For each bounded selfadjoint operator $T$ in $H_{Q}$, one can consider the variational eigenvalues of the Rayleigh quotient (cf. e.g., Weinberger [19, Ch. 3].) To do so, we denote by $\mathcal{E}_{l}$ the collection of subspaces of $H$ of dimension $l$, for all nonnegative integers $l$ less or equal to the dimension of $H$. Then the variational eigenvalues of the Rayleigh quotient corresponding to $T, Q$ are defined to be the numbers

$$
\begin{aligned}
\alpha^{(l)}[Q, T] & \equiv \sup _{E \in \mathcal{E}_{l}} \inf _{u \in E \backslash\{0\}} \frac{Q[T u, u]}{\|u\|_{Q}^{2}}, \\
\beta^{(l)}[Q, T] & \equiv \inf _{E \in \mathcal{E}_{l}} \sup _{u \in E \backslash\{0\}} \frac{Q[T u, u]}{\|u\|_{Q}^{2}},
\end{aligned}
$$

for each $1 \leq l \in \mathbb{N}$ such that $l \leq \operatorname{dim}(H)$. In Section 2, we observe that a Lipschitz continuity result holds for the dependence of $\alpha^{(l)}, \beta^{(l)}$ upon $(Q, T)$ (see Corollary 2.1.) We emphasize that despite the name, the eigenvalues of the Rayleigh quotient are only 'candidate' eigenvalues in general. For a discussion on this topic, we refer the reader to Weinberger [19, p. 45]. Next we further assume that $T$ is both compact and selfadjoint. Then the (nonzero) eigenvalues of $T$ are delivered by the variational eigenvalues of the Rayleigh quotient. By exploiting the Kato integral formula for the projection onto the eigenspaces (cf. Kato [9, (6.19) p. 178]), and by our inequalities for the variational eigenvalues, we prove a Lipschitz type inequality for the dependence of the projections upon variation of $Q, T$ (cf. Theorem 2.6). With this respect, we note that a Lipschitz continuity property for the variational eigenvalues upon variation of the operator with a fixed scalar product in $H$ is already known (see Cox [4], Gohberg and Goldberg [7, p. 123].)

Next we apply our abstract results to a concrete situation. We shall consider the dependence of the eigenvalues of the Rayleigh quotient and of the projections onto the eigenspaces of the Laplace operator $-\Delta$ with Dirichlet boundary conditions upon domain perturbation. We fix a connected open subset $\Omega$ of $\mathbb{R}^{n}$ such that the Poincaré inequality holds in $\Omega$, and we parametrize our perturbed 
domain by a homeomorphism $\phi$ of $\Omega$ onto $\phi(\Omega) \subseteq \mathbb{R}^{n}$. In Continuum Mechanics, $\phi$ plays the role of deformation of the body $\Omega$. We shall assume that $\phi$ has bounded distributional derivatives, and that the absolute value $|\operatorname{det}(D \phi)|$ of the the Jacobian determinant of $\phi$ has a strictly positive essential infimum. Then we consider the eigenvalue problem

$$
-\Delta v=\lambda v \quad \text { in } \phi(\Omega)
$$

with Dirichlet boundary conditions. Problem (2) has been defined on the $\phi$ dependent domain $\phi(\Omega)$, and we shall transform it into a problem on $\Omega$. To do so, we consider the Sobolev space $W_{0}^{1,2}(\Omega)$ obtained by taking the closure of the space $\mathcal{D}(\Omega)$ of the $C^{\infty}$ functions with compact support in $\Omega$ in the Sobolev space $W^{1,2}(\Omega)$ of distributions in $\Omega$ which have weak derivatives up to the first order in $L^{2}(\Omega)$, endowed with its usual norm (cf. (18)). By the Poincaré inequality, one has an equivalent norm in $W_{0}^{1,2}(\Omega)$ by taking the energy norm $\left\{\int_{\Omega}|D u|^{2} d x\right\}^{1 / 2}$ for all $u \in W_{0}^{1,2}(\Omega)$. We denote by $w_{0}^{1,2}(\Omega)$ the space $W_{0}^{1,2}(\Omega)$ with the scalar product associated to the energy norm. The space $w_{0}^{1,2}(\Omega)$ will play the role of our 'environment' space $H$. Then we introduce the 'variable' scalar product

$$
Q_{\phi}\left[u_{1}, u_{2}\right] \equiv \int_{\phi(\Omega)} D\left(u_{1} \circ \phi^{(-1)}\right) D\left(u_{2} \circ \phi^{(-1)}\right)^{t} d y
$$

for all $u_{1}, u_{2} \in W_{0}^{1,2}(\Omega)$, and we denote by $w_{0, \phi}^{1,2}(\Omega)$ the space $W_{0}^{1,2}(\Omega)$ endowed with the scalar product $Q_{\phi}$. Under our assumptions on $\phi$, the Poincaré inequality holds in $\phi(\Omega)$, and the function $u$ belongs to $w_{0}^{1,2}(\Omega)$ if and only if the function $u \circ \phi^{(-1)}$ belongs to $w_{0}^{1,2}(\phi(\Omega))$. Thus, as is well known, the operator $-\Delta$ is an isomorphism of $W_{0}^{1,2}(\phi(\Omega))$ onto its dual space $W^{-1,2}(\phi(\Omega))$. Since $L^{2}(\phi(\Omega))$ is naturally included in $W^{-1,2}(\phi(\Omega))$, and $w_{0}^{1,2}(\phi(\Omega))$ is imbedded into $L^{2}(\phi(\Omega))$, it can be shown that for all $u \in w_{0}^{1,2}(\Omega)$, there exists one and only one element $T_{\phi} u \in w_{0}^{1,2}(\Omega)$ such that $\left(T_{\phi} u\right) \circ \phi^{(-1)} \in w_{0}^{1,2}(\phi(\Omega))$ and

$$
-\Delta\left(\left(T_{\phi} u\right) \circ \phi^{(-1)}\right)=u \circ \phi^{(-1)} \quad \text { in } \phi(\Omega) .
$$

Clearly, problem (2) is equivalent to the eigenvalue problem

$$
T_{\phi} u=\lambda^{-1} u
$$

where $u=v \circ \phi$. Hence, we can consider the operator $T_{\phi}$ of $w_{0, \phi}^{1,2}(\Omega)$ to itself. In fact, one can easily show that $T_{\phi}$ is bounded and selfadjoint in $w_{0, \phi}^{1,2}(\Omega)$ and that the variational eigenvalues $\alpha^{(l)}\left[Q_{\phi}, T_{\phi}\right]$ coincide with the reciprocals of the Dirichlet eigenvalues of the Rayleigh quotient

$$
R_{\phi(\Omega)}(v) \equiv \frac{\int_{\phi(\Omega)}|D v|^{2} d y}{\int_{\phi(\Omega)}|v|^{2} d y}
$$


(cf. formulas (22), (25).) Instead, one cannot expect that $T_{\phi}$ be selfadjoint in $w_{0}^{1,2}(\Omega)$. Thus it becomes clear that if one wishes to preserve selfadjointness, then one has to consider different scalar products in $w_{0}^{1,2}(\Omega)$ for different $\phi$ 's. We will take $w_{0}^{1,2}(\Omega)$ as our environment space $H$, and $w_{0, \phi}^{1,2}(\Omega)$ will play the role of $H_{Q}$. By applying the abstract inequality of Corollary 2.1, we shall prove a Lipschitz type inequality relating the reciprocals of the Dirichlet eigenvalues of the Rayleigh quotient $R_{\phi(\Omega)}(v)$ on $\phi(\Omega)$ with the reciprocals of the Dirichlet eigenvalues of the Rayleigh quotient $R_{\tilde{\phi}(\Omega)}(\tilde{v})$ on $\tilde{\phi}(\Omega)$, where $\tilde{\phi}$ is another homeomorphism satisfying the same assumptions of $\phi$ (cf. Theorem 3.15). We also provide some explicit information on the Lipschitz constant, and we point out that it can be chosen to be independent of the index which enumerates the eigenvalues. We note that the inequalities relating the eigenvalues of the Rayleigh quotient on $\phi(\Omega)$ assume a simpler form in case we choose $\phi$ to be a conformal transformation in $\mathbb{R}^{2}$.

Also, we mention that Hölder type inequalities for the dependence of the eigenvalues of the Neumann Laplacian upon perturbation of the domain have been found recently by Burenkov and Davies [2], who have exploited a completely different method.

Then we assume that $\Omega$ has a finite measure, and we consider the zeta function $Z[\phi](s) \equiv \sum_{j=1}^{\infty} \lambda_{j}^{-s}[\phi]$ for $s>\frac{n}{2}$ (cf. Minakshisundaram and Pleijel [10]), and we show that if $\alpha \in] 0,1]$, and $s>\frac{n}{2}+\alpha$, then the dependence of $Z[\phi](s)$ upon $\phi$ satisfies a Hölder inequality with exponent $\alpha$ (cf. Theorem 3.20).

Finally, we assume that $\Omega$ is such that the imbedding of $W_{0}^{1,2}(\Omega)$ in $L^{2}(\Omega)$ is compact. Under such an assumption, the eigenvalues of the Rayleigh quotient exhaust all the spectrum of $-\Delta$ and have finite multiplicity. Then we deduce a Lipschitz continuity result for the projections onto the eigenspaces by exploiting our abstract results of Section 2 and the estimates for the operator $T_{\phi}$ of Proposition 3.13.

We mention that the use of transplantation, i.e., the use of a change of variables to convert a problem in a deformed domain $\phi(\Omega)$ into one in a reference domain $\Omega$, in matters involving eigenvalues for the Laplace equation goes back to Pólya and Schiffer [12]. One may wonder why we have chosen to transplant our problem into a problem for a selfadjoint operator in a space with a "variable' scalar product, instead of choosing a transformation into a problem for a selfadjoint operator in a space with a fixed scalar product. The point here is that our method has the advantage of requiring very little regularity on the transformation $\phi$.

The applications of the results of Section 2 we have presented in this paper concern only the Laplace operator, but we point out that the same ideas could be applied to a larger class of elliptic operators.

The paper is organized as follows. Section 2 provides the abstract results 
mentioned above. Section 3 contains the applications of the results of Section 2 to the Dirichlet eigenvalue problem for the Laplace operator.

\section{Lipschitz inequalities for eigenvalues and for projections onto eigenspaces.}

We first introduce some technical preliminaries and notation. Let $\mathcal{X}, \mathcal{Y}, \mathcal{Z}$ be real Banach spaces. We endow the product $\mathcal{X} \times \mathcal{Y}$ with the product norm $\|(x, y)\|_{\mathcal{X} \times \mathcal{Y}} \equiv\|x\|_{\mathcal{X}}+\|y\|_{\mathcal{Y}}$ for all $(x, y) \in \mathcal{X} \times \mathcal{Y}$. We denote by $\mathcal{L}(\mathcal{X}, \mathcal{Y})$ the Banach space of linear and continuous maps of $\mathcal{X}$ to $\mathcal{Y}$ endowed with its usual norm of the uniform convergence on the unit sphere of $\mathcal{X}$. We denote by $\mathcal{K}(\mathcal{X}, \mathcal{Y})$ the subspace of $\mathcal{L}(\mathcal{X}, \mathcal{Y})$ of those elements which are compact, i.e., which map bounded subsets of $\mathcal{X}$ to subsets of $\mathcal{Y}$ with compact closure. We denote by $\mathcal{B}(\mathcal{X} \times \mathcal{Y}, \mathcal{Z})$ the space of the bilinear and continuous maps of $\mathcal{X} \times \mathcal{Y}$ to $\mathcal{Z}$ endowed with the norm of the uniform convergence on the cross product of the unit sphere of $\mathcal{X}$ and of the unit sphere of $\mathcal{Y}$. We say that the space $\mathcal{X}$ is continuously imbedded in the space $\mathcal{Y}$ provided that $\mathcal{X}$ is a linear subspace of $\mathcal{Y}$, and that the inclusion map is continuous. We denote by $\mathbb{Z}$ the set of integer numbers, and by $\mathbb{N}$ the set of natural numbers including 0 . The inverse function of an invertible function $f$ is denoted $f^{(-1)}$, as opposed to the reciprocal of a complex-valued function $g$, or the inverse of a matrix $A$, which are denoted $g^{-1}$ and $A^{-1}$, respectively. If $A \equiv\left(a_{r s}\right)_{r, s=1, \ldots, n}$ is an $n \times n$ matrix with real entries, we set

$$
|A| \equiv\left\{\sum_{r, s=1}^{n} a_{r s}^{2}\right\}^{\frac{1}{2}},
$$

and we denote by $A^{t}$ the transpose of $A$. If $A$ is invertible, we set $A^{-t} \equiv\left(A^{-1}\right)^{t}$. Let $(H,\langle\cdot, \cdot\rangle)$ be a real Hilbert space. Let $\|\cdot\|$ denote the norm associated to the scalar product $\langle\cdot, \cdot\rangle$ on $H$, and $\operatorname{dim}(H)$ denote the possibly infinite dimension of $H$. In the sequel, we consider also other scalar products defined on the linear space $H$. Then we denote by $H_{Q}$ the linear space $H$ endowed with the scalar product $Q$ defined on $H$. We denote by $\|\cdot\|_{Q}$ the norm associated to the scalar product $Q$ on $H$. Clearly, $Q$ and $\|\cdot\|_{Q}$ do not necessarily coincide with $\langle\cdot, \cdot\rangle$ and $\|\cdot\|$, respectively. However, we shall require the imbedding of $H_{Q}$ in $H$ to be continuous, and that the scalar product $Q$ be continuous on $H$. Thus we mention the following Lemma concerning continuous bilinear forms on $H$, whose verification is straightforward.

Lemma 2.1. Let $H$ be a real Hilbert space. Let $\eta[\cdot]$ be the map of $\mathcal{B}\left(H^{2}, \mathbb{R}\right)$ to $\mathbb{R}$ defined by

$$
\eta[B] \equiv \inf \left\{\frac{B[u, u]}{\|u\|^{2}}: u \in H \backslash\{0\}\right\}
$$


for all $B \in \mathcal{B}\left(H^{2}, \mathbb{R}\right)$. Then we have

$$
|\eta[B]| \leq\|B\|_{\mathcal{B}\left(H^{2}, \mathbb{R}\right)}, \quad\left|\eta\left[B_{1}\right]-\eta\left[B_{2}\right]\right| \leq\left\|B_{1}-B_{2}\right\|_{\mathcal{B}\left(H^{2}, \mathbb{R}\right)}
$$

for all $B, B_{1}, B_{2} \in \mathcal{B}\left(H^{2}, \mathbb{R}\right)$. In particular, the set $\left\{B \in \mathcal{B}\left(H^{2}, \mathbb{R}\right): \eta[B]>0\right\}$ is open in $\mathcal{B}\left(H^{2}, \mathbb{R}\right)$.

Since scalar products are bilinear and symmetric forms, we introduce the notation

$$
\mathcal{B}_{s}\left(H^{2}, \mathbb{R}\right) \equiv\left\{B \in \mathcal{B}\left(H^{2}, \mathbb{R}\right): B[x, y]=B[y, x] \quad \forall x, y \in H\right\} .
$$

Clearly, $\mathcal{B}_{s}\left(H^{2}, \mathbb{R}\right)$ is a closed linear subspace of $\mathcal{B}\left(H^{2}, \mathbb{R}\right)$. Then the set of coercive elements of $\mathcal{B}_{s}\left(H^{2}, \mathbb{R}\right)$ is denoted by

$$
\mathcal{Q}\left(H^{2}, \mathbb{R}\right) \equiv\left\{B \in \mathcal{B}_{s}\left(H^{2}, \mathbb{R}\right): \eta[B]>0\right\} .
$$

Now we observe that if $Q$ is a scalar product on $H$, and if the imbedding of $H_{Q}$ in $H$ is a homeomorphism, then $Q \in \mathcal{Q}\left(H^{2}, \mathbb{R}\right)$, and that conversely, if $Q \in \mathcal{Q}\left(H^{2}, \mathbb{R}\right)$, then $Q$ is a scalar product on $H$, and the identity of $H_{Q}$ in $H$ is a homeomorphism. We obviously have

$$
\eta[Q]^{\frac{1}{2}}\|u\| \leq\|u\|_{Q} \leq\|Q\|_{\mathcal{B}\left(H^{2}, \mathbb{R}\right)}^{\frac{1}{2}}\|u\|,
$$

for all $u \in H$, and for all $Q \in \mathcal{Q}\left(H^{2}, \mathbb{R}\right)$, and accordingly, $H_{Q}$ is complete and has the same topology of $H$. We also note that if $Q$ belongs to $\mathcal{Q}\left(H^{2}, \mathbb{R}\right)$, then $\mathcal{L}\left(H_{Q}, H_{Q}\right)$ equals $\mathcal{L}(H, H)$ both algebraically and topologically. Similarly, $\mathcal{K}\left(H_{Q}, H_{Q}\right)$ equals $\mathcal{K}(H, H)$ both algebraically and topologically.

Our next goal is to compare the variational eigenvalues of the Rayleigh quotient of linear and continuous operators in $H_{Q}$, for different $Q$ in $\mathcal{Q}\left(H^{2}, \mathbb{R}\right)$. Thus we set

$$
\mathcal{E}_{l} \equiv\{E \leq H: \operatorname{dim}(E)=l\},
$$

for each $l \in \mathbb{N}$ such that $1 \leq l \leq \operatorname{dim}(H)$. Now let $T \in \mathcal{L}(H, H)$ (no matter whether $T$ is selfadjoint or not). We shall consider the functionals $\alpha^{(l)}, \beta^{(l)}$ defined in (1). Then we have the following technical Lemma.

Lemma 2.2. Let $H$ be a real Hilbert space. Let $Q, \tilde{Q} \in \mathcal{Q}\left(H^{2}, \mathbb{R}\right), T, \tilde{T} \in$ $\mathcal{L}(H, H)$. Then the following inequality holds:

$$
\begin{aligned}
\mid \frac{Q[T u, u]}{\|u\|_{Q}^{2}}- & \frac{\tilde{Q}[\tilde{T} u, u]}{\|u\|_{\tilde{Q}}^{2}} \mid \\
\leq & \|Q-\tilde{Q}\|_{\mathcal{B}\left(H^{2}, \mathbb{R}\right)} \\
& \times\left(\|T\|_{\mathcal{L}(H, H)} \eta[Q]^{-1}+\|\tilde{T}\|_{\mathcal{L}(H, H)} \eta[\tilde{Q}]^{-1} \eta[Q]^{-1}\|\tilde{Q}\|_{\mathcal{B}\left(H^{2}, \mathbb{R}\right)}\right) \\
& +\|T-\tilde{T}\|_{\mathcal{L}(H, H)}\|\tilde{Q}\|_{\mathcal{B}\left(H^{2}, \mathbb{R}\right)} \eta[Q]^{-1}
\end{aligned}
$$

for all $u \in H \backslash\{0\}$. 
Proof. It suffices to note that the left hand side of (5) is less or equal to

$$
\left|\frac{(Q-\tilde{Q})[T u, u]}{\|u\|_{Q}^{2}}\right|+\left|\frac{\tilde{Q}[T u-\tilde{T} u, u]}{\|u\|_{Q}^{2}}\right|+|\tilde{Q}[\tilde{T} u, u]| \cdot\left|\frac{1}{\|u\|_{Q}^{2}}-\frac{1}{\|u\|_{\tilde{Q}}^{2}}\right|,
$$

for all $u \in H \backslash\{0\}$, and to exploit the definition of $\eta[\cdot]$.

Then we are ready to prove the following Proposition, which provides a Lipschitz type inequality for the variational eigenvalues of the Rayleigh quotient $\alpha^{(l)}$ and $\beta^{(l)}$, corresponding to the operators $T, \tilde{T}$ and scalar products $Q, \tilde{Q}$.

Proposition 2.3. Let $H$ be a real Hilbert space. Let $Q, \tilde{Q} \in \mathcal{Q}\left(H^{2}, \mathbb{R}\right)$ and $T$, $\tilde{T} \in \mathcal{L}(H, H)$. Then the following inequality holds:

$$
\begin{aligned}
& \sup \left\{\left|\alpha^{(l)}[Q, T]-\alpha^{(l)}[\tilde{Q}, \tilde{T}]\right|,\left|\beta^{(l)}[Q, T]-\beta^{(l)}[\tilde{Q}, \tilde{T}]\right|\right\} \\
& \leq\|Q-\tilde{Q}\|_{\mathcal{B}\left(H^{2}, \mathbb{R}\right)} \\
& \times\left(\|T\|_{\mathcal{L}(H, H)} \eta[Q]^{-1}+\|\tilde{T}\|_{\mathcal{L}(H, H)} \eta[\tilde{Q}]^{-1} \eta[Q]^{-1}\|\tilde{Q}\|_{\mathcal{B}\left(H^{2}, \mathbb{R}\right)}\right) \\
&+\|T-\tilde{T}\|_{\mathcal{L}(H, H)}\|\tilde{Q}\|_{\mathcal{B}\left(H^{2}, \mathbb{R}\right)} \eta[Q]^{-1}
\end{aligned}
$$

for all $l \in \mathbb{N}$ such that $1 \leq l \leq \operatorname{dim}(H)$.

Proof. To shorten our notation, we denote by $A$ the right hand side of (5). Then we have

$$
\frac{Q[T u, u]}{\|u\|_{Q}^{2}}-A \leq \frac{\tilde{Q}[\tilde{T} u, u]}{\|u\|_{\tilde{Q}}^{2}} \leq \frac{Q[T u, u]}{\|u\|_{Q}^{2}}+A .
$$

Then by taking $\sup _{E \in \mathcal{E}_{l}} \inf _{u \in E \backslash\{0\}}$ and $\inf _{E \in \mathcal{E}_{l}} \sup _{u \in E \backslash\{0\}}$ in this inequality, we conclude that (6) holds.

Then we have the following immediate Corollary.

Corollary 2.1. Let $H$ be a real Hilbert space. Let $l \in \mathbb{N}$ be such that $1 \leq l \leq$ $\operatorname{dim}(H)$. Then the maps $\alpha^{(l)}[\cdot, \cdot]$ and $\beta^{(l)}[\cdot, \cdot]$ of $\mathcal{Q}\left(H^{2}, \mathbb{R}\right) \times \mathcal{L}(H, H)$ to $\mathbb{R}$ which map an element $(Q, T)$ of $\mathcal{Q}\left(H^{2}, \mathbb{R}\right) \times \mathcal{L}(H, H)$ to $\alpha^{(l)}[Q, T]$ and to $\beta^{(l)}[Q, T]$, respectively, are Lipschitz continuous on each subset $\mathcal{A}$ of $\mathcal{Q}\left(H^{2}, \mathbb{R}\right) \times \mathcal{L}(H, H)$ such that

$$
L[\mathcal{A}] \equiv \sup _{(Q, T) \in \mathcal{A}}\left\{\eta[Q]^{-1},\|Q\|_{\mathcal{B}\left(H^{2}, \mathbb{R}\right)},\|T\|_{\mathcal{L}(H, H)}\right\}<\infty .
$$

More precisely, we have

$$
\begin{aligned}
\sup \left\{\left|\alpha^{(l)}[Q, T]-\alpha^{(l)}[\tilde{Q}, \tilde{T}]\right|,\left|\beta^{(l)}[Q, T]-\beta^{(l)}[\tilde{Q}, \tilde{T}]\right|\right\} \\
\leq\left(L[\mathcal{A}]^{2}+L[\mathcal{A}]^{4}\right)\left(\|Q-\tilde{Q}\|_{\mathcal{B}\left(H^{2}, \mathbb{R}\right)}+\|T-\tilde{T}\|_{\mathcal{L}(H, H)}\right)
\end{aligned}
$$

for all $(Q, T),(\tilde{Q}, \tilde{T}) \in \mathcal{A}$. 
We note that both in Proposition 2.3, and in Corollary 2.1, we put basically no restriction on the operator $T \in \mathcal{L}(H, H)$ involved. Since we will have to consider also the case in which $T$ is compact and selfadjoint, we now introduce some notation.

We denote by $\mathcal{K}_{s}\left(H_{Q}, H_{Q}\right)$ the space of the compact selfadjoint operators in $H_{Q}$. Namely, $\mathcal{K}_{s}\left(H_{Q}, H_{Q}\right)$ is the real Banach subspace of $\mathcal{K}\left(H_{Q}, H_{Q}\right)$ of those elements $T$ such that $Q[T u, v]=Q[u, T v]$ for all $u, v \in H_{Q}$. Clearly, $\mathcal{K}_{s}\left(H_{Q}, H_{Q}\right)$ may vary with $Q \in \mathcal{Q}\left(H^{2}, \mathbb{R}\right)$. It is well known that if $T \in$ $\mathcal{K}_{s}\left(H_{Q}, H_{Q}\right)$, the spectrum $\sigma[T]$ of $T$ is finite or countable and that each point in $\sigma[T] \backslash\{0\}$ is an eigenvalue of finite multiplicity. If $\sigma[T] \cap] 0,+\infty[\neq \emptyset$, we denote by $j^{+}[T]$ the (possibly infinite) number of elements of $\left.\sigma[T] \cap\right] 0,+\infty[$, each counted with its multiplicity, and we denote by $j^{-}[T]$ the (possibly infinite) number of elements of $\sigma[T] \cap]-\infty, 0[$, each counted with its multiplicity. We also set

$$
J^{+}[T] \equiv\left\{j \in \mathbb{Z}: 1 \leq j \leq j^{+}[T]\right\}, \quad J^{-}[T] \equiv\left\{j \in \mathbb{Z}:-j^{-}[T] \leq j \leq-1\right\} .
$$

Then there exists a uniquely determined function $j \mapsto \mu_{j}[T]$ of $J[T] \equiv J^{-}[T] \cup$ $J^{+}[T]$ to $\mathbb{R} \backslash\{0\}$ such that $j \mapsto \mu_{j}[T]$ is decreasing on $J^{-}[T]$ and on $J^{+}[T]$, and such that

$$
\sigma[T] \backslash\{0\}=\left\{\mu_{j}[T]: j \in J[T]\right\},
$$

and such that each eigenvalue is repeated as many times as its multiplicity. Then we set

$$
\mathcal{M} \equiv\left\{(Q, T) \in \mathcal{B}_{s}\left(H^{2}, \mathbb{R}\right) \times \mathcal{K}(H, H): Q[T u, v]=Q[u, T v] \forall u, v \in H\right\} .
$$

Clearly, $\mathcal{M}$ is a closed subset of $\mathcal{B}_{s}\left(H^{2}, \mathbb{R}\right) \times \mathcal{K}(H, H)$. Furthermore, the set

$$
\begin{aligned}
\mathcal{O} & \equiv \mathcal{M} \cap\left(\mathcal{Q}\left(H^{2}, \mathbb{R}\right) \times \mathcal{K}(H, H)\right) \\
& =\left\{(Q, T) \in \mathcal{Q}\left(H^{2}, \mathbb{R}\right) \times \mathcal{K}(H, H): T \in \mathcal{K}_{s}\left(H_{Q}, H_{Q}\right)\right\}
\end{aligned}
$$

is obviously open in $\mathcal{M}$. Unless otherwise specified, we think of $\mathcal{O}$ as endowed with the product norm of $\mathcal{B}_{s}\left(H^{2}, \mathbb{R}\right) \times \mathcal{K}(H, H)$.

For the eigenvalues of a compact selfadjoint operator in Hilbert space, we have the following Poincaré variational formulas.

Theorem 2.4. Let $H$ be a real Hilbert space. Let $Q \in \mathcal{Q}\left(H^{2}, \mathbb{R}\right)$. Let $T \in$ $\mathcal{K}_{s}\left(H_{Q}, H_{Q}\right)$. Then the following equalities hold:

$$
\begin{array}{ll}
\mu_{j}[T]=\alpha^{(j)}[Q, T] & \forall j \in J^{+}[T] \\
\mu_{j}[T]=\beta^{(-j)}[Q, T] & \forall j \in J^{-}[T] .
\end{array}
$$

If $j^{+}[T]<+\infty$, then $\alpha^{(j)}[Q, T] \leq 0$ for all $j>j^{+}[T]$ such that $j \leq \operatorname{dim}(H)$. If $j^{-}[T]<+\infty$, then $\beta^{(-j)}[Q, T] \geq 0$ for all $j<-j^{-}[T]$ such that $-j \leq \operatorname{dim}(H)$. 
By exploiting Proposition 2.3 and Theorem 2.4, we can prove the following Theorem, which provides a Lipschitz type inequality for the eigenvalues of compact selfadjoint operators.

Theorem 2.5. Let $H$ be a real Hilbert space. If $j \in \mathbb{Z} \backslash\{0\}$, then the set

$$
\mathcal{A}_{j} \equiv\{(Q, T) \in \mathcal{O}: j \in J[T]\}
$$

is open in $\mathcal{M}$. The function $\mu_{j}[\cdot]$ of $\mathcal{A}_{j}$ to $\mathbb{R}$ which takes $(Q, T) \in \mathcal{A}_{j}$ to $\mu_{j}[T]$ is continuous. If $\mathcal{A} \subseteq \mathcal{O}$, and if $L[\mathcal{A}]<+\infty$, then $\mu_{j}[\cdot]$ is Lipschitz continuous on $\mathcal{A}_{j} \cap \mathcal{A}$. More precisely, we have

$$
\left|\mu_{j}[T]-\mu_{j}[\tilde{T}]\right| \leq\left(L[\mathcal{A}]^{2}+L[\mathcal{A}]^{4}\right)\left(\|Q-\tilde{Q}\|_{\mathcal{B}\left(H^{2}, \mathbb{R}\right)}+\|T-\tilde{T}\|_{\mathcal{L}(H, H)}\right)
$$

for all $(Q, T),(\tilde{Q}, \tilde{T}) \in \mathcal{A}_{j} \cap \mathcal{A}$. In particular, one can choose a Lipschitz constant for $\mu_{j}[\cdot]$ on $\mathcal{A}_{j} \cap \mathcal{A}$ which does not depend on $j$.

Proof. Let $j \in \mathbb{Z} \backslash\{0\}$. Let $(\tilde{Q}, \tilde{T}) \in \mathcal{A}_{j}$. We consider the case $j \in J_{\tilde{Q}}^{+}[\tilde{T}]$. Case $j \in J^{-}[\tilde{T}]$ can be treated similarly. Then we have $\mu_{j}[\tilde{T}]=\alpha^{(j)}[\tilde{Q}, \tilde{T}]>0$. Since $\mathcal{O}$ is open in $\mathcal{M}$ and $\alpha^{(j)}[\cdot, \cdot]$ is continuous on $\mathcal{O}$, then there exists a neighborhood $\mathcal{W}$ of $(\tilde{Q}, \tilde{T})$ in $\mathcal{O}$ such that $\alpha^{(j)}[\cdot, \cdot]>0$ on $\mathcal{W}$. Since $\alpha^{(1)} \geq \ldots \geq \alpha^{(j)}>0$ on $\mathcal{W}$, then Theorem 2.4 implies that $j \in J^{+}[T]$ for all $(Q, T) \in \mathcal{W}$. Thus $\mathcal{W} \subseteq \mathcal{A}_{j}$, and $\mathcal{A}_{j}$ is open in $\mathcal{M}$. Then both the continuity of $\mu_{j}[\cdot]$ on $\mathcal{W}$ and the Lipschitz continuity statement above are immediate consequences of Corollary 2.1.

We now consider the problem of the dependence of the projections onto the eigenspaces of $T$ upon variation of $T$ itself. Let $F$ be a finite subset of $\mathbb{Z} \backslash\{0\}$. We shall consider the set of pairs $(Q, T)$ for which $F \subseteq J[T]$ and for which the eigenvalues $\mu_{j}[T]$ for $j \in F$ do not equal any of the eigenvalues $\mu_{l}[T]$ of $T$ for $l$ in $(\mathbb{Z} \backslash\{0\}) \backslash F$. Thus we now introduce the following notation:

$$
\mathcal{A}[F] \equiv\left\{(Q, T) \in \mathcal{O}: F \subseteq J[T], \mu_{l}[T] \notin\left\{\mu_{j}[T]: j \in F\right\} \quad \forall l \in J[T] \backslash F\right\} .
$$

Now let $(Q, T) \in \mathcal{A}[F]$. Then we define the orthogonal projection $P_{F}[Q, T]$ of $H_{Q}$ with the scalar product $Q$ onto the subspace $E[T, F]$ generated by the subset

$$
\left\{u \in H_{Q}: T u=\mu u \text { for some } \mu \in\left\{\mu_{j}[T]: j \in F\right\}\right\}
$$

of $H_{Q}$. Then we have the following assertion.

Theorem 2.6. Let $H$ be a real Hilbert space. Let $F$ be a finite nonempty subset of $\mathbb{Z} \backslash\{0\}$. Then the map $P_{F}[\cdot, \cdot]$ of $\mathcal{A}[F]$ to $\mathcal{L}(H, H)$ which takes $(Q, T)$ to $P_{F}[Q, T]$ is continuous. Let for $(Q, T) \in \mathcal{A}[F]$

$$
\begin{aligned}
F^{*}[T] & \equiv\{l \in J[T]:\{l, l+1, l-1\} \cap F \neq \emptyset\} \\
d[T] & \equiv \min \left\{\min _{j \in F, l \in F^{*} \backslash F}\left\{\left|\mu_{j}[T]-\mu_{l}[T]\right|\right\}, \inf _{j \in F}\left|\mu_{j}[T]\right|\right\}
\end{aligned}
$$


with the understanding that the term $\min _{j \in F, l \in F^{*} \backslash F}\left\{\left|\mu_{j}[T]-\mu_{l}[T]\right|\right\}$ is omitted if $F^{*} \backslash F=\emptyset$. Then there exists a function $\Gamma$ of $] 0,+\infty[\times[0,+\infty[$ to $\mathbb{R}$ such that $\Gamma\left(\gamma_{1}, \gamma_{2}\right) \leq \Gamma\left(\delta_{1}, \delta_{2}\right)$ whenever $\left.\left(\gamma_{1}, \gamma_{2}\right),\left(\delta_{1}, \delta_{2}\right) \in\right] 0,+\infty[\times[0,+\infty[$ satisfy the inequality $\gamma_{1} \geq \delta_{1}, \gamma_{2} \leq \delta_{2}$, and such that

$$
\begin{aligned}
\| P_{F}[Q, T] & -P_{F}[\tilde{Q}, \tilde{T}] \|_{\mathcal{L}(H, H)} \\
& \leq \Gamma\left(\inf _{(Q, T) \in \mathcal{A}} d[T], L[\mathcal{A}]\right)\left(\|Q-\tilde{Q}\|_{\mathcal{B}\left(H^{2}, \mathbb{R}\right)}+\|T-\tilde{T}\|_{\mathcal{L}(H, H)}\right)
\end{aligned}
$$

for all $(Q, T),(\tilde{Q}, \tilde{T}) \in \mathcal{A}$, and for all subsets $\mathcal{A}$ of $\mathcal{A}[F]$ such that $L[\mathcal{A}]<+\infty$ and $\inf _{(Q, T) \in \mathcal{A}} d[T]>0$.

Proof. First we set $F^{+} \equiv\{j \in F: j>0\}, F^{-} \equiv\{j \in F: j<0\}$. Then $\mathcal{A}[F]=\mathcal{A}\left[F^{+}\right] \cap \mathcal{A}\left[F^{-}\right]$, and $E\left[T, F^{+}\right]$and $E\left[T, F^{-}\right]$are mutually orthogonal in $H_{Q}$, and thus we have $P_{F}[Q, T]=P_{F^{+}}[Q, T]+P_{F^{-}}[Q, T]$ for all $(Q, T) \in \mathcal{A}[F]$ (cf. e.g., Taylor and Lay [17, Thm. 12.8, Ch. IV].) Then we consider $P_{F^{+}}[Q, T]$. Indeed, $P_{F^{-}}[Q, T]$ can be treated similarly. Thus we assume $F^{+} \neq \emptyset$. Next, we split $F^{+}$into sets of consecutive indices. Thus we write $F^{+}=\cup_{r=0}^{m} F_{r}$, where each $F_{r}$ is nonempty, and contains all the indices between its minimum and its maximum, i.e., $F_{r}=\mathbb{N} \cap\left[\min F_{r}, \max F_{r}\right]$. Moreover, we assume that $\max F_{r}<\min F_{r+1}$, for $r=0, \ldots, m-1$. In particular, we have $l \notin F$ for $l \in\left\{\min F_{r}-1, \max F_{r}+1\right\}$, for all $r=0, \ldots, m$. Then $\mathcal{A}\left[F^{+}\right]=\cap_{r=0}^{m} \mathcal{A}\left[F_{r}\right]$, and the spaces $E\left[T, F_{r}\right]$ for $r=0, \ldots, m$ are mutually orthogonal in $H_{Q}$, and thus we have $P_{F^{+}}[Q, T]=\sum_{r=0}^{m} P_{F_{r}}[Q, T]$ for all $(Q, T) \in \mathcal{A}[F]$ (cf. e.g., Taylor and Lay [17, Thm. 12.8, Ch. IV].) Thus it suffices to prove the theorem for each $P_{F_{r}}$. Our next goal is to provide a lower bound for the distance of $\left\{\mu_{j}[T]: j \in F_{r}\right\}$ from the rest of the spectrum of $T$ for all $(Q, T) \in \mathcal{A}[F]$. Then we set

$d_{r}[T] \equiv \min \left\{\left|\mu_{\max F_{r}}[T]-\mu_{\max F_{r}+1}[T]\right|,\left|\mu_{\min F_{r}-1}[T]-\mu_{\min F_{r}}[T]\right|, \mu_{\max F_{r}}[T]\right\}$

with the understanding that if $\max F_{r}+1 \notin J[T]$ and $\min F_{r}-1 \notin J[T]$, then the term $\left|\mu_{\max F_{r}}[T]-\mu_{\max F_{r}+1}[T]\right|$ and $\left|\mu_{\min F_{r}-1}[T]-\mu_{\min F_{r}}[T]\right|$ do not appear in the definition of $d_{r}[T]$, respectively. Clearly, the interval $\left[\mu_{\max F_{r}}[T]-\right.$ $\left.\frac{1}{2} d_{r}[T], \mu_{\min F_{r}}[T]+\frac{1}{2} d_{r}[T]\right]$ contains $\left\{\mu_{j}[T]: j \in F_{r}\right\}$, and no other point of the spectrum of $T$, and has center $c_{r}[T] \equiv \frac{1}{2}\left(\mu_{\min F_{r}}[T]+\mu_{\max F_{r}}[T]\right)$, and halflength $\rho_{r}[T]$ equal to $\frac{1}{2} d_{r}[T]+\frac{1}{2}\left(\mu_{\min F_{r}}[T]-\mu_{\max F_{r}}[T]\right)$. Now, we consider the circle in the complex plane centered at $c_{r}[T]$ and of radius $\rho_{r}[T]$ parametrized by

$$
\gamma_{r}[T](\theta) \equiv c_{r}[T]+\rho_{r}[T] e^{i \theta}, \quad \theta \in[0,2 \pi]
$$

Clearly,

$$
\left|\gamma_{r}[T](\theta)-\xi\right| \geq \frac{1}{2} d_{r}[T] \quad \forall \xi \in \sigma[T], \forall \theta \in[0,2 \pi]
$$


Next, we introduce the complexified space $\hat{H}$ of $H$, and the complexified operator $\hat{T}$ of $T$ defined by $\hat{T}[x+i y] \equiv T[x]+i T[y]$ for all $x, y \in H$, and the complexified scalar product $\hat{Q}$ on $\hat{H}$ defined by

$$
\hat{Q}\left[x_{1}+i y_{1}, x_{2}+i y_{2}\right]=Q\left[x_{1}, x_{2}\right]+Q\left[y_{1}, y_{2}\right]+i\left(Q\left[y_{1}, x_{2}\right]-Q\left[x_{1}, y_{2}\right]\right)
$$

for all $x_{1}, x_{2}, y_{1}, y_{2} \in H$. Then by Kato [9, pp. 178, 276, 277], we have

$$
P_{F_{r}}[Q, T]=\operatorname{Re}\left\{-\frac{1}{2 \pi i} \int_{\gamma_{r}[T]}(\hat{T}-\xi \hat{I})^{(-1)} d \xi\right\}
$$

for all $(Q, T) \in \mathcal{A}[F]$, where $\hat{I}$ denotes the identity operator in $\hat{H}$. Since $T$ is selfadjoint with respect to $Q$, the operator $\hat{T}$ is selfadjoint with respect to $\hat{Q}$, and the spectrum of $\hat{T}$ coincides with $\sigma[T]$. Then, as is well known (cf. e.g., Taylor and Lay [17, Thm. 3.1, Ch. VI]), we have

$$
\left\|(\hat{T}-\xi \hat{I})^{(-1)}\right\|_{\mathcal{L}_{\mathbb{C}}(\hat{H}, \hat{H})} \leq \frac{2}{d_{r}[T]}, \quad \xi \in\left\{\gamma_{r}[T](\theta): \theta \in[0,2 \pi]\right\},
$$

where $\mathcal{L}_{\mathbb{C}}(\hat{H}, \hat{H})$ denotes the Banach space of continuous complex-linear maps of $\hat{H}$ to itself. Then we have the inequality

$$
\begin{aligned}
\| P_{F_{r}}[Q, T]- & P_{F_{r}}[\tilde{Q}, \tilde{T}] \|_{\mathcal{L}(H, H)} \\
\leq & \| \int_{0}^{2 \pi}\left[\left(\hat{T}-\gamma_{r}[T](\theta) \hat{I}\right)^{(-1)} \rho_{r}[T]\right. \\
& \left.-\left(\hat{\tilde{T}}-\gamma_{r}[\tilde{T}](\theta) \hat{I}\right)^{(-1)} \rho_{r}[\tilde{T}]\right] \frac{e^{i \theta}}{2 \pi} d \theta \|_{\mathcal{L}_{\mathbb{C}}(\hat{H}, \hat{H})} \\
\leq & \frac{2}{d_{r}[T]}\left|\rho_{r}[T]-\rho_{r}[\tilde{T}]\right|+\rho_{r}[\tilde{T}] \frac{4}{d_{r}[T] d_{r}[\tilde{T}]} \\
& \times\left(\|T-\tilde{T}\|_{\mathcal{L}(H, H)}+\left|c_{r}[T]-c_{r}[\tilde{T}]\right|+\left|\rho_{r}[T]-\rho_{r}[\tilde{T}]\right|\right)
\end{aligned}
$$

for all $(Q, T),(\tilde{Q}, \tilde{T}) \in \mathcal{A}[F]$, and for all $r=0, \ldots, m$. Now, we note that

$$
\begin{aligned}
\left|\rho_{r}[T]-\rho_{r}[\tilde{T}]\right| \leq & \frac{1}{2}\left|d_{r}[T]-d_{r}[\tilde{T}]\right| \\
& +\frac{1}{2}\left|\mu_{\max F_{r}}[T]-\mu_{\max F_{r}}[\tilde{T}]\right|+\frac{1}{2}\left|\mu_{\min F_{r}}[T]-\mu_{\min F_{r}}[\tilde{T}]\right| \\
\left|c_{r}[T]-c_{r}[\tilde{T}]\right| \leq & \frac{1}{2}\left|\mu_{\max F_{r}}[T]-\mu_{\max F_{r}}[\tilde{T}]\right|+\frac{1}{2}\left|\mu_{\min F_{r}}[T]-\mu_{\min F_{r}}[\tilde{T}]\right| \\
\left|d_{r}[T]-d_{r}[\tilde{T}]\right| \leq & 2\left|\mu_{\max F_{r}}[T]-\mu_{\max F_{r}}[\tilde{T}]\right|+\left|\mu_{\max F_{r}+1}[T]-\mu_{\max F_{r}+1}[\tilde{T}]\right| \\
& +\left|\mu_{\min F_{r}-1}[T]-\mu_{\min F_{r}-1}[\tilde{T}]\right|+\left|\mu_{\min F_{r}}[T]-\mu_{\min F_{r}}[\tilde{T}]\right| \\
\rho_{r}[\tilde{T}] \leq & \mu_{1}[\tilde{T}] \leq\|\tilde{Q}\|_{\mathcal{B}\left(H^{2}, \mathbb{R}\right)} \mid \tilde{T} \|_{\mathcal{L}(H, H)} \eta^{-1}[\tilde{Q}] \leq L^{3}[\mathcal{A}] \\
\min \left\{d_{r}[T], d_{r}[\tilde{T}]\right\} & \geq \inf _{(Q, T) \in \mathcal{A}} d[T]
\end{aligned}
$$


for all $(Q, T),(\tilde{Q}, \tilde{T}) \in \mathcal{A}$, and for all $r=0, \ldots, m$, and with the understanding that the absolute value of terms containing eigenvalues indexed by indices not in $J[T]$ should be omitted. Then by inequalities (10) and (17), we deduce the existence of $\Gamma$. The continuity is then a consequence of the existence of $\Gamma$ and of the continuous dependence of the eigenvalues of $T$ upon $T$ (cf. Theorem 2.5.)

\section{Applications to the Dirichlet eigenvalue problem for the Laplace operator}

In this section, we consider the dependence of the Dirichlet eigenvalues of the Laplace operator upon perturbation of the domain of definition. Let $\Omega$ be an open subset of $\mathbb{R}^{n}$. Throughout this section, we shall consider only the case $n \geq 2$. We denote by $L^{2}(\Omega)$ the space of square summable real valued measurable functions defined on $\Omega$ endowed with its usual norm. We denote by $W^{1,2}(\Omega)$ the Sobolev space of distributions in $\Omega$ which have weak derivatives up to the first order in $L^{2}(\Omega)$, endowed with the norm defined by

$$
\|u\|_{W^{1,2}(\Omega)} \equiv\left\{\|u\|_{L^{2}(\Omega)}^{2}+\sum_{l=1}^{n}\left\|u_{x_{l}}\right\|_{L^{2}(\Omega)}^{2}\right\}^{\frac{1}{2}}
$$

for all $u \in W^{1,2}(\Omega)$. We denote by $W_{0}^{1,2}(\Omega)$ the closure in $W^{1,2}(\Omega)$ of the space $\mathcal{D}(\Omega)$ of the real valued $C^{\infty}$ functions with compact support in $\Omega$. Now, we are interested in open connected subsets $\Omega$ of $\mathbb{R}^{n}$ for which the Poincaré inequality holds, i.e., for which the Poincaré constant $c[\Omega]$ is finite, i.e., for which

$$
c[\Omega] \equiv \sup \left\{\frac{\int_{\Omega}|u|^{2} d x}{\int_{\Omega}|D u|^{2} d x}: u \in W_{0}^{1,2}(\Omega) \backslash\{0\}\right\}^{\frac{1}{2}}<\infty .
$$

Let $\Omega$ be an open connected subset of $\mathbb{R}^{n}$ such that the Poincaré inequality holds. Then the bilinear map $\langle\cdot, \cdot\rangle$ of $\left(W_{0}^{1,2}(\Omega)\right)^{2}$ to $\mathbb{R}$ defined by

$$
\left\langle u_{1}, u_{2}\right\rangle \equiv \int_{\Omega} D u_{1} D u_{2}^{t} d x, \quad u_{1}, u_{2} \in W_{0}^{1,2}(\Omega)
$$

is a scalar product on $W_{0}^{1,2}(\Omega)$ which induces a norm equivalent to that of (18). We shall denote by $w_{0}^{1,2}(\Omega)$ the Hilbert space $W_{0}^{1,2}(\Omega)$ endowed with the scalar product of $(20)$. The strong dual $w^{-1,2}(\Omega) \equiv\left(w_{0}^{1,2}(\Omega)\right)^{\prime}$ of $w_{0}^{1,2}(\Omega)$ coincides with the strong dual $W^{-1,2}(\Omega) \equiv\left(W_{0}^{1,2}(\Omega)\right)^{\prime}$ of $W_{0}^{1,2}(\Omega)$ both algebraically and topologically. We shall always consider $w^{-1,2}(\Omega)$ as endowed with the norm

$$
\|F\|_{w^{-1,2}(\Omega)} \equiv \sup _{0 \neq u \in w_{0}^{1,2}(\Omega)} \frac{|F(u)|}{\|u\|_{w_{0}^{1,2}(\Omega)}},
$$


for all $F \in w^{-1,2}(\Omega)$, where

$$
\|u\|_{w_{0}^{1,2}(\Omega)} \equiv\left\{\int_{\Omega}|D u|^{2} d x\right\}^{\frac{1}{2}}, \quad u \in w_{0}^{1,2}(\Omega),
$$

defines the 'energy' norm associated to the scalar product in (20). Then we have the following well known result (cf. e.g., Nečas [11, Ch. 1]).

Proposition 3.1. Let $\Omega$ be an open connected subset of $\mathbb{R}^{n}$ such that the Poincaré inequality holds. For each $F$ in $W^{-1,2}(\Omega)$ there exists a unique $u \in W_{0}^{1,2}(\Omega)$ such that $\Delta u=F$ (in the sense of distributions), and

$$
\|u\|_{w^{1,2}(\Omega)} \leq\|F\|_{w^{-1,2}(\Omega)} .
$$

In particular, $-\Delta$ is a linear homeomorphism of $W_{0}^{1,2}(\Omega)$ onto $W^{-1,2}(\Omega)$.

Now we introduce the following definition.

Definition 3.2. Let $\Omega$ be a nonempty connected open subset of $\mathbb{R}^{n}$ for which the Poincaré inequality holds. Let $\mathcal{E}_{j}(\Omega)$ be the set of subspaces of $w_{0}^{1,2}(\Omega)$ of finite dimension $j, j \in \mathbb{N} \backslash\{0\}$. Then the $j$-th variational eigenvalue of the Rayleigh quotient of the Dirichlet Laplacian in $\Omega$ is the number

$$
\lambda_{j}[\Omega] \equiv \inf _{E \in \mathcal{E}_{j}(\Omega)} \sup _{u \in E \backslash\{0\}} \frac{\int_{\Omega}|D u|^{2} d x}{\int_{\Omega}|u|^{2} d x}, \quad j \in \mathbb{N} \backslash\{0\} .
$$

By the definition of the Poincaré constant $c[\Omega]$, we have

$$
\lambda_{j}[\Omega] \in\left[c^{-2}[\Omega], \infty[, \quad j \in \mathbb{N} \backslash\{0\} .\right.
$$

However, we note that the numbers $\lambda_{j}[\Omega]$ are not 'proper' eigenvalues of $-\Delta$, while they become 'proper' eigenvalues of $-\Delta$ if $W_{0}^{1,2}(\Omega)$ is compactly imbedded in $L^{2}(\Omega)$. Then we mention the following well known result.

Theorem 3.3. Let $\Omega \neq \emptyset$ be an open connected subset of $\mathbb{R}^{n}$ such that $W_{0}^{1,2}(\Omega)$ is compactly imbedded into $L^{2}(\Omega)$. Then the Poincaré constant $c[\Omega]$ is finite.

For a proof, we refer to the argument of Evans [6, Proof of Thm. 1, p. 275]. We also mention the following, which shows that $W_{0}^{1,2}(\Omega)$ is compactly imbedded into $L^{2}(\Omega)$ for sets $\Omega$ of finite measure, with no assumption whatsoever on the regularity of the boundary (cf. e.g., Tartar [18, p. 45].)

Theorem 3.4. Let $\Omega$ be an open connected subset of $\mathbb{R}^{n}$ of finite measure. Then $W_{0}^{1,2}(\Omega)$ is compactly imbedded into $L^{2}(\Omega)$. 
Now we shall consider perturbations of $\Omega$ in the form of homeomorphic images $\phi(\Omega)$ of $\Omega$ by some homeomorphism $\phi$ of $\Omega$ onto $\phi(\Omega)$ such that the Poincaré inequality still holds in $\phi(\Omega)$. Then it makes sense to consider the variational Dirichlet eigenvalues $\left\{\lambda_{j}[\phi(\Omega)]\right\}_{j \in \mathbb{N} \backslash\{0\}}$ of $-\Delta$ in the perturbed domain $\phi(\Omega)$. To simplify our notation, we shall write

$$
\lambda_{j}[\phi] \equiv \lambda_{j}[\phi(\Omega)]=\inf _{E \in \mathcal{E}_{j}(\phi(\Omega))} \sup _{v \in E \backslash\{0\}} \frac{\int_{\phi(\Omega)}|D v|^{2} d y}{\int_{\phi(\Omega)}|v|^{2} d y}, \quad j \in \mathbb{N} \backslash\{0\} .
$$

We are interested in inequalities relating $\lambda_{j}[\phi]$ corresponding to different $\phi$ 's, and we plan to obtain such inequalities by applying the results of the previous section. To do so, we first exploit a standard procedure to convert the eigenvalue problem for the Laplace operator $-\Delta$ in $\Omega$, into an eigenvalue equation in $w_{0}^{1,2}(\Omega)$ for a compact selfadjoint operator. Thus we mention the following known Lemma.

Lemma 3.5. Let $\Omega$ be an open connected subset of $\mathbb{R}^{n}$. Then the following statements hold:

(i) Let the Poincaré inequality hold in $\Omega$. Let $\mathcal{I}$ be the imbedding of $W_{0}^{1,2}(\Omega)$ into $L^{2}(\Omega)$. Let $\mathcal{J}$ be the canonical inclusion of $L^{2}(\Omega)$ into $W^{-1,2}(\Omega)$. Then the equation

$$
-\Delta u=\lambda u
$$

for $u \in W_{0}^{1,2}(\Omega), \lambda>0$ is equivalent to the equation

$$
u=-\lambda \Delta^{(-1)} \circ \mathcal{J} \circ \mathcal{I}[u]
$$

for $u \in W_{0}^{1,2}(\Omega), \lambda>0$. Both equation (23) and equation (24) have solutions $u \neq 0$ only for $\lambda>0$. The operator $T \equiv-\Delta^{(-1)} \circ \mathcal{J} \circ \mathcal{I}$ is selfadjoint in $w_{0}^{1,2}(\Omega)$. The variational eigenvalues of the Rayleigh quotient of the Dirichlet Laplacian in $\Omega$ satisfy the equality

$$
\lambda_{j}[\Omega]=\left\{\alpha^{(j)}[<\cdot, \cdot>, T]\right\}^{-1}, \quad j \in \mathbb{N} \backslash\{0\},
$$

where $\alpha^{(j)}$ is as in (1) with $H=w_{0}^{1,2}(\Omega)$ and $\langle\cdot, \cdot\rangle$ as in $(20)$.

(ii) Let $W_{0}^{1,2}(\Omega)$ be compactly imbedded into $L^{2}(\Omega)$. Then $T$ is compact (and positive definite), $J^{+}[T]=\mathbb{N} \backslash\{0\}, J^{-}[T]=\emptyset$ and $T$ has a decreasing sequence $\left\{\mu_{j}[T]\right\}_{j \in \mathbb{N} \backslash\{0\}}$ of positive eigenvalues. Accordingly, equation (23) has an increasing sequence of eigenvalues $\left\{\mu_{j}^{-1}[T]\right\}_{j \in \mathbb{N} \backslash\{0\}}$ which by virtue of Theorem 2.4 and statement (i), enjoy the variational characterization $\mu_{j}^{-1}[T]=\lambda_{j}[\Omega]$ for all $j \in \mathbb{N} \backslash\{0\}$.

Thus we will now consider equation $(24)$ on $\phi(\Omega)$ for a suitable homeomorphism $\phi$, and we will refer to $-\Delta^{(-1)}, \mathcal{J}, \mathcal{I}$ on $\phi(\Omega)$. Accordingly, we must 
impose conditions on $\phi$ so as to guarantee that the Poincaré inequality still holds in $\phi(\Omega)$, and that we can change the variables in equation $(24)$ in $\phi(\Omega)$ in order to transform it into a problem in $\Omega$. To do so, we now introduce the following class of functions $\phi$.

Definition 3.6. Let $\Omega$ be an open subset of $\mathbb{R}^{n}$. Then we set

$$
\begin{aligned}
L^{1, \infty}(\Omega) & \equiv\left\{f \in L_{\mathrm{loc}}^{1}(\Omega): \frac{\partial f}{\partial x_{r}} \in L^{\infty}(\Omega) \forall r=1, \ldots, n\right\} \\
\Phi(\Omega) & \equiv\left\{\phi \in\left(L^{1, \infty}(\Omega)\right)^{n}: \begin{array}{l}
\text { the continuous representative of } \\
\phi \text { is injective, ess } \inf _{\Omega}|\operatorname{det} D \phi|>0
\end{array}\right\},
\end{aligned}
$$

where $L_{\text {loc }}^{1}(\Omega)$ denotes the space of (equivalence classes of) locally summable measurable functions in $\Omega$, and $L^{\infty}(\Omega)$ denotes the space of (equivalence classes of) essentially bounded measurable functions.

We now collect in the following Proposition some technical properties of the functions $\phi$ in the class $\Phi(\Omega)$, which we need in the sequel. As usual, we shall denote by $\|u\|_{L^{\infty}(\Omega)}$ the essential supremum of the absolute value of $u \in L^{\infty}(\Omega)$.

Proposition 3.7. Let $\Omega$ be an open subset of $\mathbb{R}^{n}$. Then the following statements hold:

(i) If $\phi \in \Phi(\Omega)$, then $\phi(\Omega)$ is open and $\phi$ is a homeomorphism of $\Omega$ onto $\phi(\Omega)$.

(ii) If $\phi \in \Phi(\Omega)$, then $\phi$ maps sets of measure zero to sets of measure zero.

(iii) Let $\phi \in \Phi(\Omega)$. Let $v$ be a measurable function of $\phi(\Omega)$ to $\mathbb{R}$. Then $v \in$ $L^{1}(\phi(\Omega))$ if and only if $v(\phi)|\operatorname{det} D \phi| \in L^{1}(\Omega)$, and in case such membership holds, we have

$$
\int_{\phi(\Omega)} v(y) d y=\int_{\Omega} v(\phi(x))|\operatorname{det} D \phi(x)| d x .
$$

(iv) If $\phi \in \Phi(\Omega)$, then $\phi^{(-1)}$ belongs to $\Phi(\phi(\Omega))$, and $D\left(\phi^{(-1)}\right)$ coincides with the inverse matrix $\left(D \phi\left(\phi^{(-1)}\right)\right)^{-1}$.

(v) Let $\phi \in \Phi(\Omega)$. If $u \in L^{2}(\Omega)$, then the composite function $u \circ \phi^{(-1)}$ belongs to $L^{2}(\phi(\Omega))$. The operator $C_{\phi^{(-1)}}$ of $L^{2}(\Omega)$ to $L^{2}(\phi(\Omega))$ defined by $C_{\phi^{(-1)}}[u] \equiv u \circ \phi^{(-1)}$ for all $u \in L^{2}(\Omega)$, is a linear homeomorphism. The inverse of $C_{\phi(-1)}$ coincides with the operator $C_{\phi}$ defined by $C_{\phi}[v] \equiv v \circ \phi$ for all $v \in L^{2}(\phi(\Omega))$.

(vi) If $\phi \in \Phi(\Omega)$ and $v \in W^{1,2}(\phi(\Omega))$, then $D(v \circ \phi)=D v(\phi) D \phi$.

(vii) If $\phi \in \Phi(\Omega)$, then the operator $C_{\phi^{(-1)}}$ restricts a linear homeomorphism of $W^{1,2}(\Omega)$ onto $W^{1,2}(\phi(\Omega))$, and of $W_{0}^{1,2}(\Omega)$ onto $W_{0}^{1,2}(\phi(\Omega))$. Furthermore, $W_{0}^{1,2}(\Omega)$ is compactly imbedded into $L^{2}(\Omega)$ if and only if $W_{0}^{1,2}(\phi(\Omega))$ is compactly imbedded into $L^{2}(\phi(\Omega))$. 
(viii) If $\phi \in \Phi(\Omega)$, then

$$
c[\phi(\Omega)] \leq\left\{\frac{\|\operatorname{det} D \phi\|_{L^{\infty}(\Omega)}}{\operatorname{essinf}_{\Omega}|\operatorname{det} D \phi|}\right\}^{\frac{1}{2}}\||D \phi|\|_{L^{\infty}(\Omega)} c[\Omega] .
$$

(ix) If $\Omega$ has finite measure, and if $\phi \in \Phi(\Omega)$, then $\phi(\Omega)$ has finite measure.

Proof. Statement (i) is well known to hold (cf. e.g., Deimling [5, p. 23].) Statement (ii) follows by Reshetnyak [14, Cor. 1, p. 182]. Statement (iii) holds by Reshetnyak [14, Thm. 2.2, p. 99].

Since the elements of $\Phi(\Omega)$ are quasiconformal in the sense of Reshetnyak $[14$, p. 62 , Lemma 6.7 p. 190] on each connected component of $\Omega$, then the inverse map of $\phi$ is also quasiconformal and the formula for the derivatives of $\phi^{(-1)}$ holds (cf. Reshetnyak [14, Thm. 8.3 p. 215].) Hence, statement (iv) holds.

We now prove $(\mathrm{v})$. Let $u \in L^{2}(\Omega)$. Since $\phi$ maps sets of measure zero to sets of measure zero, $u \circ \phi^{(-1)}$ is measurable on $\phi(\Omega)$, and we have $\int_{\phi(\Omega)} \mid u \circ$ $\left.\phi^{(-1)}\right|^{2} d y=\int_{\Omega}|u|^{2}|\operatorname{det} D \phi| d x$. Since $|\operatorname{det} D \phi|$ is essentially bounded and has a positive essential infimum, the operator $C_{\phi^{(-1)}}$ is continuous with its inverse. We now show that $C_{\phi(-1)}$ is surjective. Let $v \in L^{2}(\phi(\Omega))$. By (ii) and (iv), $v \circ \phi$ is measurable in $\Omega$. By (iii), we have $\int_{\phi(\Omega)}|v|^{2} d y=\int_{\Omega}|v \circ \phi|^{2}|\operatorname{det} D \phi| d x$. Then $v \circ \phi \in L^{2}(\Omega)$, and $C_{\phi^{(-1)}}[v \circ \phi]=v$.

Statement (vi) can be verified by exploiting the well known density of $C^{\infty}(\phi(\Omega)) \cap W^{1,2}(\phi(\Omega))$ in $W^{1,2}(\phi(\Omega))$, statement (iii) and Reshetnyak [14, p. 23].

We now prove statement (vii). By statement (v), the operator $C_{\phi^{(-1)}}$ is a homeomorphism of $L^{2}(\Omega)$ onto $L^{2}(\phi(\Omega))$. If $u \in W^{1,2}(\Omega)$, then $u \circ \phi^{(-1)} \in$ $L^{2}(\phi(\Omega))$. By (iv) and (vi), it follows that

$$
D\left(u \circ \phi^{(-1)}\right)=D u\left(\phi^{(-1)}\right)\left(D \phi\left(\phi^{(-1)}\right)\right)^{-1} .
$$

Then by Hölder inequality, and by the essential boundedness of $\left(D \phi\left(\phi^{(-1)}\right)\right)^{-1}$, and by the continuity of $C_{\phi^{(-1)}}$ from $L^{2}(\Omega)$ to $L^{2}(\phi(\Omega))$, we can easily deduce that $C_{\phi^{(-1)}}$ is linear and continuous from $W^{1,2}(\Omega)$ to $W^{1,2}(\phi(\Omega))$. By the Open Mapping Theorem, we can conclude that $C_{\phi^{(-1)}}$ is a linear homeomorphism onto $W^{1,2}(\phi(\Omega))$, provided that we show the surjectivity of $C_{\phi^{(-1)}}$ onto $W^{1,2}(\phi(\Omega))$. Now let $v \in W^{1,2}(\phi(\Omega))$. As we have seen, $C_{\phi(-1)}[v \circ \phi]=v$ and $v \circ \phi \in L^{2}(\Omega)$. By (vi), we have $D(v \circ \phi)=D v(\phi) D \phi$. Then by Hölder inequality, and by the essential boundedness of $D \phi$, and by the inclusion $C_{\phi}\left(L^{2}(\phi(\Omega))\right) \subseteq L^{2}(\Omega)$, we deduce that $v \circ \phi \in W^{1,2}(\Omega)$. Thus $C_{\phi^{(-1)}}$ is surjective. The elements of $C_{\phi^{(-1)}}[\mathcal{D}(\Omega)]$ have compact support in $\phi(\Omega)$ and belong to $W^{1,2}(\phi(\Omega))$. Thus by a standard convolution argument with a family of mollifiers, we deduce that $C_{\phi^{(-1)}}[\mathcal{D}(\Omega)] \subseteq W_{0}^{1,2}(\phi(\Omega))$. Since $W_{0}^{1,2}(\phi(\Omega))$ is a closed subspace of 
$W^{1,2}(\phi(\Omega))$, the definition of $W_{0}^{1,2}(\Omega)$ and the continuity of $C_{\phi^{(-1)}}$ imply that $C_{\phi^{(-1)}}\left[W_{0}^{1,2}(\Omega)\right] \subseteq W_{0}^{1,2}(\phi(\Omega))$. Similarly, we can prove that $C_{\phi}=\left(C_{\phi^{(-1)}}\right)^{(-1)}$ maps $W_{0}^{1,2}(\phi(\Omega))$ to $W_{0}^{1,2}(\Omega)$.

We now prove statement (viii). By statements (iii), (iv) and (vi), we have

$$
\begin{aligned}
c[\phi(\Omega)]^{2} & =\sup _{0 \neq v \in W_{0}^{1,2}(\phi(\Omega))} \frac{\int_{\phi(\Omega)}|v|^{2} d y}{\int_{\phi(\Omega)}|D v|^{2} d y} \\
& \leq \frac{\|\operatorname{det} D \phi\|_{L^{\infty}(\Omega)}}{\operatorname{essinf}_{\Omega}|\operatorname{det} D \phi|} \sup _{0 \neq v \in W_{0}^{1,2}(\phi(\Omega))} \frac{\int_{\Omega}|v \circ \phi|^{2} d x}{\int_{\Omega}\left|D(v \circ \phi)(D \phi)^{-1}\right|^{2} d x} .
\end{aligned}
$$

Since the matrix $D \phi$ is invertible almost everywhere in $\Omega$, we have the inequality $\left|D(v \circ \phi)(D \phi)^{-1}\right|^{2} \geq|D(v \circ \phi)|^{2}|(D \phi)|^{-2}$. Hence, the inequality of statement (viii) holds.

Statement (ix) is an immediate consequence of statement (iii) and of the essential boundedness of $|\operatorname{det} D \phi|$.

We now deduce from Proposition 3.7 the validity of the following, which collects some elementary properties of the bilinear form $Q_{\phi}$.

Proposition 3.8. Let $\Omega$ be an open subset of $\mathbb{R}^{n}$ for which the Poincaré inequality holds. Then the following statements hold:

(i) Let $\phi \in \Phi(\Omega)$. The function $Q_{\phi}$ of $\left(W_{0}^{1,2}(\Omega)\right)^{2}$ to $\mathbb{R}$ defined by (3) satisfies

$$
Q_{\phi}\left[u_{1}, u_{2}\right]=\int_{\Omega} D u_{1}(D \phi)^{-1}(D \phi)^{-t} D u_{2}^{t}|\operatorname{det} D \phi| d x, \quad u_{1}, u_{2} \in W_{0}^{1,2}(\Omega) .
$$

The function $Q_{\phi}$ is a scalar product in $W_{0}^{1,2}(\Omega)$ which makes $W_{0}^{1,2}(\Omega)$ a Hilbert space denoted by the symbol $w_{0, \phi}^{1,2}(\Omega)$. The operator $C_{\phi^{(-1)}}$ restricts a surjective isometry of $w_{0, \phi}^{1,2}(\Omega)$ onto $w_{0}^{1,2}(\phi(\Omega))$. Finally, if $u \in W_{0}^{1,2}(\Omega)$, then

$$
\begin{aligned}
\frac{\operatorname{essinf}_{\Omega}^{\frac{1}{2}}|\operatorname{det} D \phi|}{\||D \phi|\|_{L^{\infty}(\Omega)}}\|u\|_{w_{0}^{1,2}(\Omega)} & \leq\|u\|_{w_{0, \phi}^{1,2}(\Omega)} \\
& \leq\|\operatorname{det} D \phi\|_{L^{\infty}(\Omega)}^{\frac{1}{2}}\left\|\mid(D \phi)^{-1}\right\|_{L^{\infty}(\Omega)}\|u\|_{w_{0}^{1,2}(\Omega)} .
\end{aligned}
$$

(ii) If $\phi \in \Phi(\Omega)$, then

$$
\frac{\operatorname{essinf}_{\Omega}|\operatorname{det} D \phi|}{\||D \phi|\|_{L^{\infty}(\Omega)}^{2}} \leq \eta\left[Q_{\phi}\right] \leq\left\|\left|(D \phi)^{-1}\right|\right\|_{L^{\infty}(\Omega)}^{2}\|\operatorname{det} D \phi\|_{L^{\infty}(\Omega)},
$$

where

$$
\eta\left[Q_{\phi}\right]=\inf \left\{\frac{\int_{\Omega}\left|D u(D \phi)^{-1}\right|^{2}|\operatorname{det} D \phi| d x}{\int_{\Omega}|D u|^{2} d x}: u \in W_{0}^{1,2}(\Omega) \backslash\{0\}\right\} .
$$


(iii) If $\phi, \tilde{\phi} \in \Phi(\Omega)$, then

$$
\begin{aligned}
& \left\|Q_{\phi}-Q_{\tilde{\phi}}\right\|_{\mathcal{B}\left(\left(w_{0}^{1,2}(\Omega)\right)^{2}, \mathbb{R}\right)} \\
& \quad \leq\left\|\left|(D \phi)^{-1}(D \phi)^{-t}\right| \operatorname{det} D \phi\left|-(D \tilde{\phi})^{-1}(D \tilde{\phi})^{-t}\right| \operatorname{det} D \tilde{\phi}||\right\|_{L^{\infty}(\Omega)}
\end{aligned}
$$

Proof. Proposition 3.7 and a straightforward verification shows that $Q_{\phi}$ is a scalar product and that the formula in (i) holds. Then we note that the inequality of (i) and statement (ii) follow by the inequality $|D u|^{2} \leq\left|D u(D \phi)^{-1}\right|^{2}|D \phi|^{2}$. By Proposition 3.7 (vii), $C_{\phi^{(-1)}}$ is a bijection of $W_{0}^{1,2}(\Omega)$ onto $W_{0}^{1,2}(\phi(\Omega))$. Then by definition of $Q_{\phi}$, we have $\int_{\phi(\Omega)}\left|D\left(u \circ \phi^{(-1)}\right)\right|^{2} d y=Q_{\phi}[u, u]$, and statement (i) follows. Statement (iii) is an obvious consequence of statement (i).

Then we can introduce the following operator.

Definition 3.9. Let $\Omega$ be an open connected subset of $\mathbb{R}^{n}$ for which the Poincaré inequality holds. Let $\phi \in \Phi(\Omega)$. Then $T_{\phi}$ denotes the operator of $W_{0}^{1,2}(\Omega)$ to itself defined by setting

$$
T_{\phi}[u] \equiv-C_{\phi} \circ \Delta^{(-1)} \circ \mathcal{J} \circ \mathcal{I} \circ C_{\phi^{(-1)}}[u]
$$

for all $u \in W_{0}^{1,2}(\Omega)$. Here $-\Delta^{(-1)}, \mathcal{J}, \mathcal{I}$ are relative to $\phi(\Omega)$.

Then we have the following result.

Theorem 3.10. Let $\Omega$ be an open connected subset of $\mathbb{R}^{n}$ for which the Poincaré inequality holds. Let $\phi \in \Phi(\Omega)$. Then the following statements hold.

(i) $T_{\phi}$ is a selfadjoint operator of $w_{0, \phi}^{1,2}(\Omega)$ to itself.

(ii) It holds

$$
\lambda_{j}[\phi]=\left\{\alpha^{(j)}\left[Q_{\phi}, T_{\phi}\right]\right\}^{-1}, \quad j \in \mathbb{N} \backslash\{0\},
$$

where $\alpha^{(j)}$ is as in (1) with $H=w_{0}^{1,2}(\Omega)$.

(iii) If $(\lambda, v) \in \mathbb{R} \times\left(W_{0}^{1,2}(\phi(\Omega)) \backslash\{0\}\right)$ satisfies

$$
v=-\lambda \Delta^{(-1)} \circ \mathcal{J} \circ \mathcal{I}[v]
$$

then $\lambda>0$, and $\left(\mu \equiv \lambda^{-1}, u \equiv v \circ \phi\right)$ belongs to $] 0,+\infty\left[\times W_{0}^{1,2}(\Omega)\right.$ and satisfies the equation

$$
\mu u=T_{\phi} u .
$$

Conversely, if $(\mu, u) \in \mathbb{R} \times\left(W_{0}^{1,2}(\Omega) \backslash\{0\}\right)$ satisfies equation (27), then $\mu>0$, and $\left(\lambda \equiv \mu^{-1}, v \equiv u \circ \phi^{(-1)}\right)$ belongs to $\mathbb{R} \times\left(W_{0}^{1,2}(\phi(\Omega)) \backslash\{0\}\right)$ and satisfies equation (26). 
(iv) If we further assume that the imbedding of $W_{0}^{1,2}(\Omega)$ into $L^{2}(\Omega)$ is compact, then $T_{\phi}$ is also compact and we have $J^{+}\left[T_{\phi}\right]=\mathbb{N} \backslash\{0\}, J^{-}\left[T_{\phi}\right]=\emptyset$, and equation (27) has a decreasing sequence $\left\{\mu_{j}[\phi]\right\}_{j \in \mathbb{N} \backslash\{0\}}$ of eigenvalues in ] $0,+\infty\left[\right.$, and $\mu_{j}[\phi]=\lambda_{j}^{-1}[\phi]$.

Proof. We first verify that $T_{\phi}$ is selfadjoint in $w_{0, \phi}^{1,2}(\Omega)$. Let $u_{1}, u_{2} \in w_{0, \phi}^{1,2}(\Omega)$. Then $u_{1} \circ \phi^{(-1)}, u_{2} \circ \phi^{(-1)} \in w_{0}^{1,2}(\phi(\Omega))$, and

$$
\begin{aligned}
Q_{\phi}\left[T_{\phi} u_{1}, u_{2}\right] & =\int_{\phi(\Omega)} D\left(\left(T_{\phi} u_{1}\right) \circ \phi^{(-1)}\right) D\left(u_{2} \circ \phi^{(-1)}\right)^{t} d y \\
& =-\int_{\phi(\Omega)} D\left(\Delta^{(-1)} \circ \mathcal{J} \circ \mathcal{I}\left[u_{1} \circ \phi^{(-1)}\right]\right) D\left(u_{2} \circ \phi^{(-1)}\right)^{t} d y \\
& =\int_{\phi(\Omega)} u_{1} \circ \phi^{(-1)} u_{2} \circ \phi^{(-1)} d y .
\end{aligned}
$$

Then the symmetry of $Q_{\phi}$ implies selfadjointness. Statement (ii) follows by the definition of $\lambda_{j}[\phi]$, by (21), (28) and by Proposition 3.8 (i), and by the definition of $Q_{\phi}, T_{\phi}$. We now prove statement (iii). If the pair $(\lambda, v) \in \mathbb{R} \times$ $\left(W_{0}^{1,2}(\phi(\Omega)) \backslash\{0\}\right)$ solves $(26)$, then $\lambda>0$ by Lemma 3.5 , and $\left(\mu \equiv \lambda^{-1}, u \equiv\right.$ $v \circ \phi)$ belongs to $] 0,+\infty\left[\times W_{0}^{1,2}(\Omega)\right.$ by Proposition 3.7. Then the pair $(u, \mu)$ solves (27) by the definition of $T_{\phi}$. Statement (iv) is an obvious consequence of statement (iii), of the compactness of $\mathcal{I}$, and of Lemma 3.5.

In order to perform the necessary estimates on $T_{\phi}$ and on $T_{\phi}-T_{\tilde{\phi}}$, we find convenient to rewrite $T_{\phi}$ in a different way. Thus we now introduce two auxiliary operators.

Definition 3.11. Let $\Omega$ be an open subset of $\mathbb{R}^{n}$. Let $\phi \in \Phi(\Omega)$. Then we define the following two operators.

(i) Let $\mathcal{J}_{\phi}$ be the operator of $L^{2}(\Omega)$ to $W^{-1,2}(\Omega)$ defined by

$$
\mathcal{J}_{\phi}[u][w] \equiv \int_{\Omega} u w|\operatorname{det} D \phi| d x, \quad w \in W_{0}^{1,2}(\Omega) .
$$

(ii) Let $\Delta_{\phi}$ be the operator of $W_{0}^{1,2}(\Omega)$ to $W^{-1,2}(\Omega)$ defined by

$$
\Delta_{\phi}[u] \equiv C_{\phi^{(-1)}}^{t} \circ \Delta \circ C_{\phi^{(-1)}}[u], \quad u \in W_{0}^{1,2}(\Omega),
$$

where $C_{\phi^{(-1)}}^{t}$ denotes the transpose of the operator $C_{\phi^{(-1)}}$ of $W_{0}^{1,2}(\Omega)$ to $W_{0}^{1,2}(\phi(\Omega))$ (cf. Proposition 3.7.)

Then we have the following properties. 
Proposition 3.12. Let $\Omega$ be an open connected subset of $\mathbb{R}^{n}$ for which the Poincaré inequality holds. Then the following statements hold.

(i) $\mathcal{J}_{\phi}$ is linear and continuous, and

$$
\left\|\mathcal{J}_{\phi} \circ \mathcal{I}\right\|_{\mathcal{L}\left(w_{0}^{1,2}(\Omega), w^{-1,2}(\Omega)\right)} \leq c^{2}[\Omega]\|\operatorname{det} D \phi\|_{L^{\infty}(\Omega)} .
$$

(ii) The operator $\Delta_{\phi}$ is a linear homeomorphism of $W_{0}^{1,2}(\Omega)$ onto $W^{-1,2}(\Omega)$. Furthermore,

$$
\Delta_{\phi}[u][w]=-\int_{\Omega} D u(D \phi)^{-1}(D \phi)^{-t} D w^{t}|\operatorname{det} D \phi| d x,
$$

for all $u, w \in W_{0}^{1,2}(\Omega)$.

(iii) The following diagram commutes:

$$
\begin{array}{ccccccc}
W_{0}^{1,2}(\phi(\Omega)) & \Delta^{-\Delta^{(-1)}} & W^{-1,2}(\phi(\Omega)) & \stackrel{\mathcal{J}}{\leftrightarrows} & L^{2}(\phi(\Omega)) & \stackrel{\mathcal{I}}{ } & W_{0}^{1,2}(\phi(\Omega)) \\
C_{\phi} \downarrow & & C_{\phi}^{t} \uparrow & & C_{\phi^{(-1)}} \uparrow & & C_{\phi^{(-1)}} \uparrow \\
W_{0}^{1,2}(\Omega) & \stackrel{-\Delta_{\phi}^{(-1)}}{\longleftarrow} & W^{-1,2}(\Omega) & \stackrel{\mathcal{J}_{\phi}}{\longleftarrow} & L^{2}(\Omega) & \stackrel{\mathcal{I}}{ } & W_{0}^{1,2}(\Omega)
\end{array}
$$

In particular, $T_{\phi}=-\Delta_{\phi}^{(-1)} \circ \mathcal{J}_{\phi} \circ \mathcal{I}$.

Proof. Statement (i) is an immediate consequence of the essential boundedness of $|\operatorname{det} D \phi|$, of the Hölder inequality, and of the Poincaré inequality (19). The continuity of $\Delta_{\phi}$ follows by the continuity of $C_{\phi^{(-1)}}^{t}, \Delta, C_{\phi^{(-1)}}$. By Proposition 3.7, $C_{\phi^{(-1)}}$ is a linear homeomorphism of $W_{0}^{1,2}(\Omega)$ onto $W_{0}^{1,2}(\phi(\Omega))$. Hence, $C_{\phi^{(-1)}}^{t}$ is also a linear homeomorphism. By Proposition 3.1, $\Delta$ is a homeomorphism of $W_{0}^{1,2}(\phi(\Omega))$ onto $W^{-1,2}(\phi(\Omega))$. Thus $\Delta_{\phi}$ is a linear homeomorphism. By changing the variable in the integral corresponding to the weak formulation of the definition of $\Delta$, we immediately deduce the validity of (31). We now prove that the diagram in (iii) commutes. Equalities $C_{\phi}^{t} \circ \mathcal{J}_{\phi}=\mathcal{J} \circ C_{\phi^{(-1)}}$ and $C_{\phi^{(-1)}} \circ \mathcal{I}=\mathcal{I} \circ C_{\phi^{(-1)}}$ are of immediate verification. Finally, equality $\Delta_{\phi}^{(-1)}=C_{\phi} \circ \Delta^{(-1)} \circ C_{\phi}^{t}$ follows by the definition of $\Delta_{\phi}$, and by the obvious equality $C_{\phi^{(-1)}}=\left(C_{\phi}\right)^{(-1)}$.

We are now ready to prove the inequalities we need on $T_{\phi}, T_{\phi}-T_{\tilde{\phi}}$.

Proposition 3.13. Let $\Omega$ be an open connected subset of $\mathbb{R}^{n}$ for which the Poincaré inequality holds. Then the following statements hold:

(i) If $\phi \in \Phi(\Omega)$, then

$$
\begin{aligned}
& \left\|T_{\phi}\right\|_{\mathcal{L}\left(w_{0}^{1,2}(\Omega), w_{0}^{1,2}(\Omega)\right)} \\
& \quad \leq c^{2}[\phi(\Omega)]\left(\frac{\|\operatorname{det} D \phi\|_{L^{\infty}(\Omega)}}{\operatorname{essinf}_{\Omega}|\operatorname{det} D \phi|}\right)^{\frac{1}{2}}\||D \phi|\|_{L^{\infty}(\Omega)}\left\|\left|(D \phi)^{-1}\right|\right\|_{L^{\infty}(\Omega)} .
\end{aligned}
$$


(ii) If $\phi, \tilde{\phi} \in \Phi(\Omega)$, then

$$
\begin{aligned}
\| T_{\phi}- & T_{\tilde{\phi}} \|_{\mathcal{L}\left(w_{0}^{1,2}(\Omega), w_{0}^{1,2}(\Omega)\right)} \\
\leq & c^{2}[\Omega] \frac{\||D \phi|\|_{L^{\infty}(\Omega)}^{2}}{\operatorname{essinf}_{\Omega}|\operatorname{det} D \phi|}\left\{\||\operatorname{det} D \phi|-|\operatorname{det} \tilde{D} \tilde{\phi}|\|_{L^{\infty}(\Omega)}\right. \\
& +\left\|\left|(D \phi)^{-1}(D \phi)^{-t}\right| \operatorname{det} D \phi\left|-(D \tilde{\phi})^{-1}(D \tilde{\phi})^{-t}\right| \operatorname{det} D \tilde{\phi}||\right\|_{L^{\infty}(\Omega)} \\
& \left.\times \frac{\|\operatorname{det} D \tilde{\phi}\|_{L^{\infty}(\Omega)}}{\operatorname{essinf}_{\Omega}|\operatorname{det} D \tilde{\phi}|}\||D \tilde{\phi}|\|_{L^{\infty}(\Omega)}^{2}\right\}
\end{aligned}
$$

Proof. If $u \in W_{0}^{1,2}(\Omega)$, then by Propositions 3.1 and 3.8 (i) we have

$$
\begin{aligned}
\left\|T_{\phi} u\right\|_{w_{0}^{1,2}(\Omega)} & \leq \frac{\||D \phi|\|_{L^{\infty}(\Omega)}}{\operatorname{essinf}_{\Omega}^{1 / 2}|\operatorname{det} D \phi|}\left\|T_{\phi} u\right\|_{w_{0, \phi}^{1,2}(\Omega)} \\
& =\frac{\||D \phi|\|_{L^{\infty}(\Omega)}}{\operatorname{essinf}_{\Omega}^{1 / 2}|\operatorname{det} D \phi|}\left\|\left(T_{\phi} u\right) \circ \phi^{(-1)}\right\|_{w_{0}^{1,2}(\phi(\Omega))} \\
& \leq \frac{\||D \phi|\|_{L^{\infty}(\Omega)}}{\operatorname{essinf}_{\Omega}^{1 / 2}|\operatorname{det} D \phi|}\left\|\mathcal{J} \circ \mathcal{I}\left[u \circ \phi^{(-1)}\right]\right\|_{w^{-1,2}(\phi(\Omega))} .
\end{aligned}
$$

By the Hölder inequality, by the definition of $c[\phi(\Omega)]$ and by Proposition 3.8 (i), we conclude that statement (i) holds. We now prove statement (ii). Let $u \in W_{0}^{1,2}(\Omega)$, then $T_{\phi} u, T_{\tilde{\phi}} u \in W_{0}^{1,2}(\Omega)$ and by Proposition 3.12 (iii), we have

$$
\begin{aligned}
& \left\|T_{\phi} u-T_{\tilde{\phi}} u\right\|_{w_{0}^{1,2}(\Omega)} \\
& \leq\left\|\Delta_{\phi}^{(-1)}\right\|_{\mathcal{L}\left(w^{-1,2}(\Omega), w_{0}^{1,2}(\Omega)\right)}\left\|\mathcal{J}_{\phi} \circ \mathcal{I}[u]-\Delta_{\phi} \circ \Delta_{\tilde{\phi}}^{(-1)} \circ \mathcal{J}_{\tilde{\phi}} \circ \mathcal{I}[u]\right\|_{w^{-1,2}(\Omega)} \\
& \leq\left\|C_{\phi}\right\|_{\mathcal{L}\left(w_{0}^{1,2}(\phi(\Omega)), w_{0}^{1,2}(\Omega)\right)}\left\|\Delta^{(-1)}\right\|_{\mathcal{L}\left(w^{-1,2}(\phi(\Omega)), w_{0}^{1,2}(\phi(\Omega))\right)} \\
& \times\left\|C_{\phi}^{t}\right\|_{\mathcal{L}\left(w^{-1,2}(\Omega), w^{-1,2}(\phi(\Omega))\right)} \\
& \times \sup _{w \in W_{0}^{1,2}(\Omega) \backslash\{0\}} \frac{\left|\left\langle\mathcal{J}_{\phi} \circ \mathcal{I}[u]-\Delta_{\phi} \circ \Delta_{\tilde{\phi}}^{(-1)} \circ \mathcal{J}_{\tilde{\phi}} \circ \mathcal{I}[u], w\right\rangle\right|}{\|w\|_{w_{0}^{1,2}(\Omega)}} .
\end{aligned}
$$

By the rule of change of variable in integrals (see Proposition 3.7 (iii)), we obtain

$$
\begin{array}{rl}
\left\|C_{\phi}\right\|^{2} & \mathcal{L}\left(w_{0}^{1,2}(\phi(\Omega)), w_{0}^{1,2}(\Omega)\right) \\
& \leq \frac{\||D \phi|\|_{L^{\infty}(\Omega)}^{2}}{\operatorname{essinf}_{\Omega}|\operatorname{det} D \phi|} \sup _{v \in W_{0}^{1,2}(\phi(\Omega)) \backslash\{0\}} \frac{\int_{\Omega}\left|D(v \circ \phi)(D \phi)^{-1}\right|^{2}|\operatorname{det} D \phi| d x}{\int_{\phi(\Omega)}|D v|^{2} d y} \\
& =\frac{\||D \phi|\|_{L^{\infty}(\Omega)}^{2}}{\operatorname{ess~inf}_{\Omega}|\operatorname{det} D \phi|}
\end{array}
$$


Moreover, by elementary functional analysis, we have

$$
\left\|C_{\phi}^{t}\right\|_{\mathcal{L}\left(w^{-1,2}(\Omega), w^{-1,2}(\phi(\Omega))\right)} \leq\left\|C_{\phi}\right\|_{\mathcal{L}\left(w_{0}^{1,2}(\phi(\Omega)), w_{0}^{1,2}(\Omega)\right)} .
$$

Then by the Hölder inequality, by the definition of the Poincaré constant and by Proposition 3.12 (i), (ii), we have

$$
\begin{aligned}
\mid\left\langle\mathcal{J}_{\phi} \circ\right. & \left.\mathcal{I}[u]-\Delta_{\phi} \circ \Delta_{\tilde{\phi}}^{(-1)} \circ \mathcal{J}_{\tilde{\phi}} \circ \mathcal{I}[u], w\right\rangle \mid \\
= & \left|\int_{\Omega} u w\right| \operatorname{det} D \phi \mid d x \\
& +\int_{\Omega} D\left(\Delta_{\tilde{\phi}}^{(-1)} \circ \mathcal{J}_{\tilde{\phi}} \circ \mathcal{I}[u]\right)(D \phi)^{-1}(D \phi)^{-t}(D w)^{t}|\operatorname{det} D \phi| d x \mid \\
\leq & \left|\int_{\Omega} u w\right| \operatorname{det} D \phi \mid d x \\
& +\int_{\Omega} D\left(\Delta_{\tilde{\phi}}^{(-1)} \circ \mathcal{J}_{\tilde{\phi}} \circ \mathcal{I}[u]\right)(D \tilde{\phi})^{-1}(D \tilde{\phi})^{-t}(D w)^{t}|\operatorname{det} D \tilde{\phi}| d x \mid \\
& +\left|\int_{\Omega} D\left(\Delta_{\tilde{\phi}}^{(-1)} \circ \mathcal{J}_{\tilde{\phi}} \circ \mathcal{I}[u]\right)(D \phi)^{-1}(D \phi)^{-t}(D w)^{t}\right| \operatorname{det} D \phi \mid d x \\
& -\int_{\Omega} D\left(\Delta_{\tilde{\phi}}^{(-1)} \circ \mathcal{J}_{\tilde{\phi}} \circ \mathcal{I}[u]\right)(D \tilde{\phi})^{-1}(D \tilde{\phi})^{-t}(D w)^{t}|\operatorname{det} D \tilde{\phi}| d x \mid \\
\leq & c^{2}[\Omega]\||\operatorname{det} D \phi|-|\operatorname{det} D \tilde{\phi}|\| L_{L^{\infty}(\Omega)}\|u\|_{w_{0}^{1,2}(\Omega)}\|w\|_{w_{0}^{1,2}(\Omega)} \\
& +\left\|\left|(D \tilde{\phi})^{-1}(D \tilde{\phi})^{-t}\right| \operatorname{det} D \tilde{\phi}\left|-(D \phi)^{-1}(D \phi)^{-t}\right| \operatorname{det} D \phi||\right\| L_{L^{\infty}(\Omega)} \\
& \times\left\|\Delta_{\tilde{\phi}}^{(-1)}\right\|\left\|_{\mathcal{L}\left(w^{-1,2}(\Omega), w_{0}^{1,2}(\Omega)\right)} c[\Omega]^{2}\right\| \operatorname{det} D \tilde{\phi}\left\|_{L^{\infty}(\Omega)}\right\| u\left\|_{w_{0}^{1,2}(\Omega)}\right\| w \|_{w_{0}^{1,2}(\Omega)}
\end{aligned}
$$

Thus by the above inequalities and by Proposition 3.1, we deduce that (32) holds.

We now give the following technical Lemma, whose verification is immediate.

Lemma 3.14. Let $\Omega$ be an open subset of $\mathbb{R}^{n}$. Let $\phi, \tilde{\phi} \in \Phi(\Omega)$. Then the following four inequalities hold:

$$
\begin{aligned}
& \|\operatorname{det} D \phi\|_{L^{\infty}(\Omega)} \leq n !\||D \phi|\|_{L^{\infty}(\Omega)}^{n} \\
& \left\|\left|(D \phi)^{-1}\right|\right\|_{L^{\infty}(\Omega)} \leq \frac{n !\||D \phi|\|_{L^{\infty}(\Omega)}^{n-1}}{\operatorname{essinf}_{\Omega}|\operatorname{det} D \phi|}
\end{aligned}
$$


and

$$
\begin{aligned}
& |\operatorname{det} D \phi-\operatorname{det} D \tilde{\phi}| \\
& \quad \leq n ! n\||D \phi-D \tilde{\phi}|\|_{L^{\infty}(\Omega)} \sup \left\{\||D \phi|\|_{L^{\infty}(\Omega)},\||D \tilde{\phi}|\|_{L^{\infty}(\Omega)}\right\}^{n-1} \\
& \quad\left\|\left|(D \phi)^{-1}-(D \tilde{\phi})^{-1}\right|\right\|_{L^{\infty}(\Omega)} \\
& \quad \leq\left\|\left|(D \phi)^{-1}\right|\right\|_{L^{\infty}(\Omega)}\left\|\left|(D \tilde{\phi})^{-1}\right|\right\|_{L^{\infty}(\Omega)}\||D \phi-D \tilde{\phi}|\|_{L^{\infty}(\Omega)} .
\end{aligned}
$$

We are now able to prove the first of our two main results. By Propositions 3.8 and 3.13, which in particular give information on the dependence upon $\phi$ of $Q_{\phi}$ and $T_{\phi}$, respectively, it turns out that our inequalities depend on the pseudometric $\delta$ on $\Phi(\Omega)$ defined by

$$
\begin{aligned}
\delta(\phi, \tilde{\phi}) \equiv & \left\{\||\operatorname{det} D \phi|-|\operatorname{det} D \tilde{\phi}|\|_{L^{\infty}(\Omega)}\right. \\
& \left.+\left\|\left|(D \phi)^{-1}(D \phi)^{-t}\right| \operatorname{det} D \phi\left|-(D \tilde{\phi})^{-1}(D \tilde{\phi})^{-t}\right| \operatorname{det} D \tilde{\phi}||\right\|_{L^{\infty}(\Omega)}\right\}
\end{aligned}
$$

for all $\phi, \tilde{\phi} \in \Phi(\Omega)$, and therefore, by Lemma 3.14 above, on $\||D \phi-D \tilde{\phi}|\|_{L^{\infty}(\Omega)}$. By combining these results with Corollary 2.1 with $H \equiv w_{0}^{1,2}(\Omega)$, Propositions 3.7, 3.8, Theorem 3.10 (ii), Proposition 3.13 and Lemma 3.14, we deduce the validity of the following statement.

Theorem 3.15. Let $n \geq 2$. Then there exist two functions $\Lambda_{1}, \Lambda_{2}$ of the set ] $0,+\infty\left[{ }^{2} \times\left[0,+\infty\left[{ }^{3}\right.\right.\right.$ to $[0,+\infty[$ such that

$$
\Lambda_{r}\left(\gamma_{1}, \ldots, \gamma_{5}\right) \leq \Lambda_{r}\left(\zeta_{1}, \ldots, \zeta_{5}\right) \quad r=1,2,
$$

whenever $\left(\gamma_{1}, \ldots, \gamma_{5}\right)$ and $\left.\left(\zeta_{1}, \ldots, \zeta_{5}\right) \in\right] 0,+\infty\left[{ }^{2} \times\left[0,+\infty\left[^{3}\right.\right.\right.$ satisfy $\gamma_{l} \geq \zeta_{l}$ for $l=1,2$, and $\gamma_{l} \leq \zeta_{l}$ for $l=3,4,5$, and such that

$$
\begin{aligned}
& \left|\lambda_{j}^{-1}[\phi]-\lambda_{j}^{-1}[\tilde{\phi}]\right| \\
& \leq \Lambda_{1}\left(\operatorname{ess} \inf _{\Omega}|\operatorname{det} D \phi|, \operatorname{ess} \inf _{\Omega}|\operatorname{det} D \tilde{\phi}|,\||D \phi|\|_{L^{\infty}(\Omega)},\right. \\
& \left.\||D \tilde{\phi}|\|_{L^{\infty}(\Omega)}, c[\Omega]\right) \delta(\phi, \tilde{\phi}) \\
& \leq \Lambda_{2}\left(\operatorname{ess} \inf _{\Omega}|\operatorname{det} D \phi|, \operatorname{ess} \inf _{\Omega}|\operatorname{det} D \tilde{\phi}|,\||D \phi|\|_{L^{\infty}(\Omega)}\right. \text {, } \\
& \left.\||D \tilde{\phi}|\|_{L^{\infty}(\Omega)}, c[\Omega]\right)\||D \phi-D \tilde{\phi}|\|_{L^{\infty}(\Omega)}
\end{aligned}
$$

for all nonempty open connected subsets $\Omega$ of $\mathbb{R}^{n}$ such that the Poincaré inequality (19) holds, and for all $\phi, \tilde{\phi} \in \Phi(\Omega)$. In particular, the functions $\Lambda_{1}$ and $\Lambda_{2}$ depend only on $n$ and the right hand side of (36) does not depend on $j \in \mathbb{N} \backslash\{0\}$. 
In particular, we note that if $\phi=R \tilde{\phi}+a$ for some $n \times n$ orthogonal matrix $R$ with real entries, and for some $a \in \mathbb{R}^{n}$, then the first part of inequality (36) implies that $\lambda_{j}[\phi]=\lambda_{j}[\tilde{\phi}]$ for all $j \in \mathbb{N} \backslash\{0\}$.

Clearly, one can consider the seminorm

$$
\|\phi\|_{\left(L^{1, \infty}(\Omega)\right)^{n}} \equiv\||D \phi|\|_{L^{\infty}(\Omega)} \quad \forall \phi \in\left(L^{1, \infty}(\Omega)\right)^{n}
$$

on $\left(L^{1, \infty}(\Omega)\right)^{n}$ which induces a pseudometric on the subset $\Phi(\Omega)$ of $\left(L^{1, \infty}(\Omega)\right)^{n}$ different from the pseudometric $\delta$ introduced in (35). Then we deduce the validity of the following immediate Corollary of Theorem 3.15.

Corollary 3.1. Let $\Omega$ be an open connected subset of $\mathbb{R}^{n}$ for which the Poincaré inequality holds. Let $\tau>0$,

$$
\Phi_{\tau}(\Omega) \equiv\left\{\phi \in \Phi(\Omega): \frac{1}{\tau} \leq \operatorname{ess} \inf _{\Omega}|\operatorname{det} D \phi|, \operatorname{ess} \sup _{\Omega}|D \phi| \leq \tau\right\} .
$$

Then for each $j \in \mathbb{N} \backslash\{0\}$, the functions $\lambda_{j}^{-1}[\cdot]$ are Lipschitz continuous from $\Phi_{\tau}(\Omega)$ endowed with the pseudometric $\delta$ of (35), or with the seminorm (37) to $\mathbb{R}$, and one can choose a Lipschitz constant depending solely on $n, c[\Omega], \tau$, and not on $j \in \mathbb{N} \backslash\{0\}$.

In the two dimensional case, we can take $\phi \in \Phi(\Omega)$ holomorphic. Then

$$
\operatorname{det} D \phi=\left|\phi^{\prime}\right|^{2}, \quad \frac{D \phi(D \phi)^{t}}{|\operatorname{det} D \phi|}=I .
$$

Thus the inequalities of Proposition 3.8, of Proposition 3.13, of Lemma 3.14, and of Theorem 3.15 assume a simplified form, which for brevity we do not report here.

We now deduce some consequences of Theorem 3.15 on the behaviour of the zeta function of the eigenvalues of the Laplace operator considered by Minakshisundaram-Pleijel [10]. To do so, we first introduce a particular case of a result of Rozenbljum [15, Thm. 1, p. 1540] on the eigenvalue distribution for the Dirichlet problem. Since we are now dealing with sets $\Omega$ of finite measure, the imbedding of $W_{0}^{1,2}(\Omega)$ in $L^{2}(\Omega)$ is well known to be compact (cf. Theorem 3.4).

Theorem 3.16. Let $\Omega$ be a nonempty open connected subset of $\mathbb{R}^{n}$ of finite measure. Then there exists a constant $c_{1}>0$ depending only on $n$, and not on $\Omega$ such that

$$
j \leq c_{1} \lambda_{j}^{\frac{n}{2}} \operatorname{meas}(\Omega)
$$

for all $j \in \mathbb{N} \backslash\{0\}$, where $\left\{\lambda_{j}\right\}_{j \in \mathbb{N} \backslash\{0\}}$ are the eigenvalues of (23).

Then by combining Theorem 3.16 with Proposition 3.7 (ix), we deduce the following. 
Proposition 3.17. Let $\Omega$ be a nonempty open connected subset of $\mathbb{R}^{n}$ of finite measure. Let $\phi \in \Phi(\Omega)$. Then for each $s>\frac{n}{2}$, the series $\sum_{j=1}^{\infty} \lambda_{j}^{-s}[\phi]$ converges. Moreover, there exists a constant $c_{1}>0$ depending only on $n$, and not on $\Omega$ such that

$$
\sum_{j=1}^{\infty} \lambda_{j}^{-s}[\phi] \leq\left(c_{1} \operatorname{meas}(\phi(\Omega))\right)^{\frac{2 s}{n}} \sum_{j=1}^{\infty} j^{-\frac{2 s}{n}}
$$

We are now ready to introduce the zeta function considered by Minakshisundaram-Pleijel.

Definition 3.18. Let $\Omega$ be a a nonempty open connected subset of $\mathbb{R}^{n}$ of finite measure. Let $\phi \in \Phi(\Omega)$. We denote by $Z[\phi]$ the function of $] \frac{n}{2},+\infty[$ to $\mathbb{R}$ defined by

$$
\left.Z[\phi](s) \equiv \sum_{j=1}^{\infty} \lambda_{j}^{-s}[\phi], \quad s \in\right] \frac{n}{2},+\infty[
$$

We now prove the following elementary technical Lemma.

Lemma 3.19. Let $\alpha \in] 0,1], s \in\left[\alpha+1,+\infty\left[\right.\right.$. Let $\left\{a_{j}\right\}_{j \in \mathbb{N}},\left\{b_{j}\right\}_{j \in \mathbb{N}}$ be two sequences in $\left[0,+\infty\left[\right.\right.$ such that both the series $\sum_{j=0}^{\infty} a_{j}^{s-\alpha}$ and $\sum_{j=0}^{\infty} b_{j}^{s-\alpha}$ are convergent. Then both the series $\sum_{j=0}^{\infty} a_{j}^{s}$ and $\sum_{j=0}^{\infty} b_{j}^{s}$ are convergent, and

$$
\left|\sum_{j=0}^{\infty} a_{j}^{s}-\sum_{j=0}^{\infty} b_{j}^{s}\right| \leq s 2^{s-\alpha-1}\left(\sum_{j=0}^{\infty} a_{j}^{s-\alpha}+\sum_{j=0}^{\infty} b_{j}^{s-\alpha}\right) \sup _{j \in \mathbb{N}}\left|a_{j}-b_{j}\right|^{\alpha} .
$$

Proof. First we note that the left hand side of (39) is less or equal to

$$
\begin{aligned}
s \sum_{j=0}^{\infty}\left(a_{j}+b_{j}\right)^{s-1}\left|a_{j}-b_{j}\right| & \leq s \sum_{j=0}^{\infty}\left(a_{j}+b_{j}\right)^{s-1}\left|a_{j}-b_{j}\right|^{\alpha}\left|a_{j}-b_{j}\right|^{1-\alpha} \\
& \leq s \sum_{j=0}^{\infty}\left(a_{j}+b_{j}\right)^{s-\alpha}\left|a_{j}-b_{j}\right|^{\alpha} .
\end{aligned}
$$

Since $\left(a_{j}+b_{j}\right)^{s-\alpha} \leq 2^{s-\alpha-1}\left(a_{j}^{s-\alpha}+b_{j}^{s-\alpha}\right)$, we conclude that (39) holds.

Then by Theorem 3.15, by Proposition 3.17 and by Lemma 3.19 we immediately deduce the validity of the following theorem. 
Theorem 3.20. Let $n \geq 2$. Let $\alpha \in] 0,1], s>\frac{n}{2}+\alpha$. Let $\Lambda_{1}$ be as in Theorem 3.15. Then the following inequality holds:

$$
\begin{aligned}
& |Z[\phi](s)-Z[\tilde{\phi}](s)| \\
& \leq s 2^{s-\alpha-1}\left(c_{1} \operatorname{meas}(\Omega)\right)^{\frac{2(s-\alpha)}{n}} \sum_{j=1}^{\infty} j^{-\frac{2(s-\alpha)}{n}}\left(\|\operatorname{det} D \phi\|_{L^{\infty}(\Omega)}^{\frac{2(s-\alpha)}{n}}+\|\operatorname{det} D \tilde{\phi}\|_{L^{\infty}(\Omega)}^{\frac{2(s-\alpha)}{n}}\right) \\
& \quad \times \Lambda_{1}^{\alpha}\left(\operatorname{ess} \inf _{\Omega}|\operatorname{det} D \phi|, \operatorname{ess} \inf _{\Omega}|\operatorname{det} D \tilde{\phi}|,\||D \phi|\|_{L^{\infty}(\Omega)},\||D \tilde{\phi}|\|_{L^{\infty}(\Omega)}, c[\Omega]\right) \\
& \quad \times \delta^{\alpha}(\tilde{\phi}, \phi)
\end{aligned}
$$

for all nonempty open connected subsets $\Omega$ of $\mathbb{R}^{n}$ of finite measure, and for all $\phi, \tilde{\phi} \in \Phi(\Omega)$.

Then by Lemma 3.14 and by the second part of inequality (36) we deduce the validity of the following immediate Corollary of Theorem 3.20.

Corollary 3.2. Let $n \geq 2$. Let $\alpha \in] 0,1], s>\frac{n}{2}+\alpha$. Let $\Omega$ be a nonempty open connected subset of $\mathbb{R}^{n}$ of finite measure. Let $\tau>0$. Let $\Phi_{\tau}(\Omega)$ be the set introduced in Corollary 3.1. Then the function $Z[\cdot](s)$ is Hölder continuous with exponent $\alpha$ from the set $\Phi_{\tau}(\Omega)$ endowed with the pseudometric $\delta$ of (35), or with the seminorm (37) to $\mathbb{R}$, and one can choose a Hölder constant depending solely on $n, \Omega, \tau, \alpha, s$.

Finally, we consider the dependence of the projections onto the eigenspaces of $-\Delta$ upon perturbation of $\phi$. Let $F$ be a finite subset of $\mathbb{N} \backslash\{0\}$. Let $\Omega$ be an open connected subset of $\mathbb{R}^{n}$ such that $W_{0}^{1,2}(\Omega)$ is compactly imbedded into $L^{2}(\Omega)$. Then we set

$$
\Phi(\Omega, F) \equiv\left\{\phi \in \Phi(\Omega): \lambda_{l}[\phi] \notin\left\{\lambda_{j}[\phi]: j \in F\right\} \quad \forall l \in \mathbb{N} \backslash(F \cup\{0\})\right\} .
$$

Now let $\phi \in \Phi(\Omega, F)$. Then we define the orthogonal projection $P_{F}[\phi]$ of $w_{0, \phi}^{1,2}(\Omega)$ onto the subspace $E[\phi, F]$ generated by the subset

$$
\left\{u \in W_{0}^{1,2}(\Omega):-\Delta_{\phi} u=\lambda \mathcal{J}_{\phi} \circ \mathcal{I}[u] \text { for some } \lambda \in\left\{\lambda_{j}[\phi]: j \in F\right\}\right\}
$$

of $w_{0, \phi}^{1,2}(\Omega)$ with the scalar product $Q_{\phi}$. Then we set

$$
F^{*} \equiv\{l \in \mathbb{N} \backslash\{0\}:\{l-1, l, l+1\} \cap F \neq \emptyset\}
$$

and

$$
\begin{aligned}
d[\phi] & \equiv \min \left\{\min _{j \in F, l \in F^{*} \backslash F}\left|\lambda_{j}^{-1}[\phi]-\lambda_{l}^{-1}[\phi]\right|, \inf _{j \in F} \lambda_{j}^{-1}[\phi]\right\} \\
& =\min _{j \in F, l \in F^{*} \backslash F}\left|\lambda_{j}^{-1}[\phi]-\lambda_{l}^{-1}[\phi]\right|
\end{aligned}
$$


for all $\phi \in \Phi(\Omega, F)$. Then by combining Theorem 2.6, Propositions 3.8, 3.13, and Lemma 3.14, we deduce the validity of the following theorem.

Theorem 3.21. Let $n \geq 2$. Let $F$ be a finite subset of $\mathbb{N} \backslash\{0\}$. Then there exist two functions $\Gamma_{1}, \Gamma_{2}$ of the set $] 0,+\infty\left[{ }^{4} \times\left[0,+\infty\left[{ }^{3}\right.\right.\right.$ to $[0,+\infty[$ such that $\Gamma_{r}\left(\gamma_{1}, \ldots, \gamma_{7}\right) \leq \Gamma_{r}\left(\zeta_{1}, \ldots, \zeta_{7}\right)$ holds whenever $\left(\gamma_{1}, \ldots, \gamma_{7}\right)$ and $\left(\zeta_{1}, \ldots, \zeta_{7}\right)$ in ] $0,+\infty\left[{ }^{4} \times\left[0,+\infty\left[{ }^{3}\right.\right.\right.$ satisfy the inequalities $\gamma_{l} \geq \zeta_{l}$ for $l=1, \ldots, 4$ and $\gamma_{l} \leq \zeta_{l}$ for $l=5,6,7$, and such that

$$
\begin{aligned}
\| P_{F}[\phi]-P_{F}[\tilde{\phi}] & \|_{\mathcal{L}\left(w_{0}^{1,2}(\Omega), w_{0}^{1,2}(\Omega)\right)} \\
\leq & \Gamma_{1}\left(\operatorname{ess} \inf _{\Omega}|\operatorname{det} D \phi|, \operatorname{ess} \inf _{\Omega}|\operatorname{det} D \tilde{\phi}|, d[\phi], d[\tilde{\phi}]\right. \\
& \left.\||D \phi|\|_{L^{\infty}(\Omega)},\||D \tilde{\phi}|\|_{L^{\infty}(\Omega)}, c[\Omega]\right) \delta(\tilde{\phi}, \phi) \\
\leq & \Gamma_{2}\left(\operatorname{ess} \inf _{\Omega}|\operatorname{det} D \phi|, \operatorname{ess} \inf _{\Omega}|\operatorname{det} D \tilde{\phi}|, d[\phi], d[\tilde{\phi}],\right. \\
& \left.\||D \phi|\|_{L^{\infty}(\Omega)},\||D \tilde{\phi}|\|_{L^{\infty}(\Omega)}, c[\Omega]\right)\||D \phi-D \tilde{\phi}|\|_{L^{\infty}(\Omega)}
\end{aligned}
$$

for all nonempty open connected subsets $\Omega$ of $\mathbb{R}^{n}$ such that $W_{0}^{1,2}(\Omega)$ is compactly imbedded into $L^{2}(\Omega)$, and for all $\phi, \tilde{\phi} \in \Phi(\Omega, F)$.

We remark that if $F$ is a finite subset of $\mathbb{N} \backslash\{0\}$, then the continuity of the dependence of the eigenvalues upon $\phi \in \Phi(\Omega, F)$ (see Theorem 3.15) and Theorem 3.21 ensure that $P_{F}[\cdot]$ satisfies a Lipschitz condition on the compact subsets of $\Phi(\Omega, F)$.

Acknowledgement. The authors are indebted to Prof. G.V. Rozenbljum for pointing out a number of references.

\section{References}

[1] Berger, M. S.: Nonlinearity and Functional Analysis. New York: Academic Press 1977.

[2] Burenkov, V. I. and E. B. Davies: Spectral stability of the Neumann Laplacian. J. Differential Equations 186 (2002), 485 - 508.

[3] Courant, R. and D. Hilbert: Methods of Mathematical Physics, Vol. I. New York: Interscience 1953.

[4] Cox, S. J.: The generalized gradient at a multiple eigenvalue. J. Functional Analysis 133 (1995), 30 - 40.

[5] Deimling, K.: Nonlinear Functional Analysis. Berlin: Springer 1985.

[6] Evans, L. C.: Partial Differential Equations. Providence: Amer. Math. Soc. 1998. 
[7] Gohberg, I. and S. Goldberg: Basic Operator Theory. Boston: Birkhäuser 1981.

[8] Henry, D.: Perturbation of the Boundary in Boundary-Value Problems of Partial Differential Equations. London Mathematical Society Lecture Notes No. 318. Cambridge: Cambridge University Press 2005.

[9] Kato, T.: Perturbation Theory for Linear Operators. Berlin - New York: Springer 1976.

[10] Minakshisundaram, S. and A. Pleijel: Some properties of the eigenfunctions of the Laplace-operator on Riemannian manifolds. Canadian J. Math. 1 (1949), $242-256$.

[11] Nečas, J.: Les Méthodes Directes en Théorie des Équations Elliptiques. Paris: Masson et Cie 1967.

[12] Pólya, G. and M. Schiffer: Convexity of functionals by transplantation. J. d'Analyse Math. 3 (1954), 245 - 346.

[13] Prodi, G.: Dipendenza dal dominio degli autovalori dell'operatore di Laplace. Istit. Lombardo Accad. Sci. Lett. Rend. A 128 (1994), 3 - 18.

[14] Reshetnyak, Yu. G.: Space Mappings with Bounded Distortion. Translations of Mathematical Monographs 73. Providence: Amer. Math. Soc. 1989.

[15] Rozenbljum, G. V.: On the distribution of eigenvalues of the first boundary value problem in unbounded regions. Sov. Math. Dokl. 12 (1971), 1539 - 1542.

[16] Sokolowski, J. and J. P. Zolésio: Introduction to Shape Optimization. Shape Sensitivity Analysis. Berlin: Springer 1992.

[17] Taylor, A. E. and D. C. Lay: Introduction to Functional Analysis. New York: John Wiley 1980.

[18] Tartar, L.: An Introduction to Sobolev Spaces and Interpolation Spaces. Pittsburgh: Carnegie-Mellon Univ. 2000.

[19] Weinberger, H. F.: Variational Methods for eigenvalue approximation. Regional Conf. Ser. Appl. Math. 15. Philadelphia: SIAM 1974.

Received 22.01.2004; in revised form 29.07.2004 